\title{
A bilevel shape optimization problem for the exterior Bernoulli free boundary value problem
}

\author{
HENRY KASUMBA \\ Johann Radon Institute for Computational and Applied Mathematics, \\ Altenbergerstraße 69, 4040 Linz, Austria \\ E-mail:henry.kasumba@oeaw.at \\ KARL KUNISCH \\ Institute for Mathematics and Scientific Computing, University of Graz, \\ Heinrichstraße 36, 8010 Graz, Austria \\ E-mail:karl.kunisch@uni-graz.at \\ ANTOINE LAURAIN \\ Department of Mathematics, Technical University of Berlin, \\ Straße des 17. Juni 136, 10623 Berlin, Germany \\ E-mail:laurain@math.tu-berlin.de
}

[Received 8 February 2013 and in revised form 13 June 2014]

\begin{abstract}
A bilevel shape optimization problem with the exterior Bernoulli free boundary problem as lowerlevel problem and the control of the free boundary as the upper-level problem is considered. Using the shape of the inner boundary as the control, we aim at reaching a specific shape for the free boundary. A rigorous sensitivity analysis of the bilevel shape optimization in the infinite-dimensional setting is performed. The numerical realization using two different cost functionals presented in this paper demonstrate the efficiency of the approach.
\end{abstract}

2010 Mathematics Subject Classification: Primary 35R35, 49Q10; Secondary 35Q93.

Keywords: Bernoulli free boundary problem; shape optimization; multilevel optimization.

\section{Introduction}

Let $\omega \subset \varepsilon$ with $\omega$ a smooth and bounded domain in $\mathbb{R}^{2}$. Further $\varepsilon$ is a bounded domain in $\mathbb{R}^{2}$ which is supposed to contain all admissible shapes and is referred to as the hold-all domain. We define the set of admissible shapes as

$$
\bigcirc_{a d}=\left\{\Omega \subset \mathbb{R}^{2} \text { a bounded domain : } \bar{\omega} \subset \Omega, \bar{\Omega} \subset \varepsilon\right\} \text {. }
$$

For given $\mu \in \mathbb{R}, \mu<0$, we consider the following free boundary problem:

$\left(\mathcal{F}_{\omega}\right)$ : Find $\Omega \in \mathcal{O}_{a d}$ such that problem (1.1)-(1.4) has a solution, 


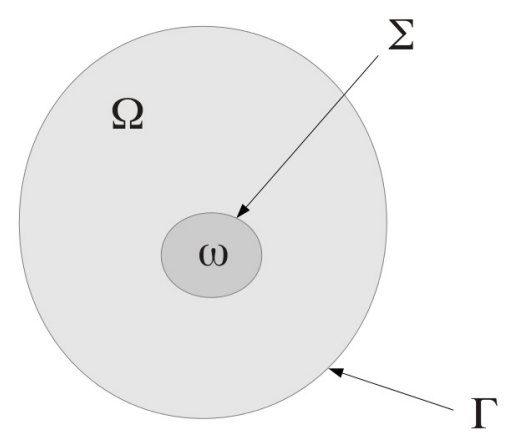

FIG. 1. The exterior Bernoulli problem

where

$$
\begin{aligned}
-\Delta u=0 & \text { in } \quad \Omega \backslash \bar{\omega}, \\
u=1 \quad \text { on } \quad \Sigma:=\partial \omega, & \text { on } \quad \Gamma:=\partial \Omega, \\
u=0 & \text { on } \quad \Gamma,
\end{aligned}
$$

with $u$ in $H_{\Gamma}^{1}(\Omega \backslash \bar{\omega})$, the standard Sobolev space of $H^{1}$-functions whose trace vanishes on $\Gamma$. This problem is known as the exterior Bernoulli free boundary problem due to $\bar{\omega} \subset \Omega$. Note that (1.1)-(1.4) is over-determined since two boundary conditions are specified on $\Gamma$, and in general does not have solutions. However for particular sets $\Omega$, or equivalently free boundaries $\Gamma$, problem (1.1)-(1.4) may have a solution. Problem (1.1)-(1.4) originates, for instance, from the description of free boundaries for ideal fluids [8, pp. 138-140]. Other applications leading to similar formulations include electrochemistry and electromagnetics [9].

Typically, the shape of $\Gamma$ is not known analytically except for some particular configurations of the inner boundary $\Sigma$. A number of authors have analyzed and solved problem $\left(\mathcal{F}_{\omega}\right)$ for a given fixed domain $\omega$, see for instance $[8, \mathrm{Ch} .3],[3,4,9,14,17]$ and references therein. We use the notation $\left(\mathcal{F}_{\omega}\right)$ to emphasize the dependence of the solution $\Gamma$ on $\omega$ as we will use $\omega$ to control $\Gamma$ in the problem considered in this paper.

The over-specification of conditions on $\Gamma$ naturally suggests to formulate (1.1)-(1.4) as an optimization problem; this approach has been used in [14] for instance. Subsequently, an interesting control problem arises when $\Sigma$ is used to control the solution $\Gamma$ of $\left(\mathfrak{F}_{\omega}\right)$. This gives rise to a bilevel shape optimization problem, where the free boundary value problem constitutes the lower-level optimization problem, and the upper-level consists in minimization with respect to $\Sigma$. A similar bilevel problem has been treated for the Bernoulli problem in [24] in the discrete setting, where a sensitivity analysis was performed for the Bernoulli problem using an automatic differentiation technique. In the present work, we carry out a rigorous sensitivity analysis of the cost functionals with respect to the control $\omega$ (or equivalently $\Sigma$ ) in the infinite-dimensional setting using the tools of shape calculus [8, 22]. For this purpose we introduce two cost functionals to drive the free set $\Omega$ as close as possible to a given desired set $E$. 
Control of problems defined on unknown domains plays a crucial role in the quality assessment of many applications such as continuous casting of steel [19], welding processes [27], thin film manufacturing processes [21], to mention but a few. In the literature on mathematical programs, these problems are referred to as multilevel optimization problems. They consist of programs which have a subset of their variables constrained to be an optimal solution of other programs. Such problems were first considered in [5]; see also [6] for a review. In shape optimization only a few bilevel problems have been considered due to their inherent difficulty. In [22, Section 4.3.2] shape controllability of the free boundary of an obstacle problem is studied. In [24, 25] shape and topology optimization of Bernoulli free boundary problems are considered. Shape optimization problems in fluid dynamics governed by free surface flows are considered in [16] where a sensitivity analysis of the free surface problem with the Navier-Stokes equations as constraints is formally studied.

Turning to numerical realization of the bilevel optimization problem, a possible approach consists in discarding one of the two boundary conditions on the free boundary and to append it to the cost functional on the upper level by using a penalty or augmented Lagrangian approach. Using this strategy, solving for the state $u$ becomes a classical linear boundary value problem with wellposed boundary data in the lower level problem. Unfortunately, as noted in [24], this approach leads to serious convergence problems. A further disadvantage that was noted in [24] is that, depending on the formulation, a locally optimal triplet $(u, \omega, \Omega)$ might not represent a physical solution to the free boundary problem. For this reason, we adopt a segregation approach to solve the optimization problem, i.e., we find a solution to the free boundary problem $\left(\mathcal{F}_{\omega}\right)$ first and then proceed to the upper level represented by the minimization of the cost functional. In this iterative procedure, $\left(\mathcal{F}_{\omega}\right)$ has to be solved several times for varying $\omega$. Therefore, one needs an efficient and robust solver for this type of problem. Possible solution strategies include trial methods, linearization methods (continuous or discrete) [7], and shape optimization methods [11]. Here we use a regularized fixed point method, which is a trial method. The main advantage of this approach is that it solves $\left(\mathcal{F}_{\omega}\right)$ using some simple updating formula based only on the solution of a state system. Moreover this method locally converges super-linearly [9].

The remainder of this paper is organized as follows. Section 2 describes the setting of the free boundary and optimization problems. The sensitivity analysis of the bilevel problems is performed in Section 3. In Section 4, the numerical algorithm used to solve the optimization problems is given. Numerical examples that support the theoretical results are then presented.

\section{Setting of the problem}

In this section the mathematical notations, the algorithm for solving the free boundary problem, and the setting of the optimization problems are presented.

\section{$2.1 \quad$ Notations}

Here we collect some notations and definitions that we need in our subsequent discussion. Throughout the paper we restrict ourselves to the two dimensional case.

Vectors: We use bold fonts for vectors $\mathbf{x}=\left(x_{1}, x_{2}\right)^{T} \in \mathbb{R}^{2}$ with norm $|\mathbf{x}|_{\mathbb{R}^{2}}=\left(\sum_{j=1}^{2} x_{j}^{2}\right)^{1 / 2}$ and vector-valued functions are also indicated by bold letters. Two notations for the inner product in $\mathbb{R}^{2}$ shall be used, namely $(x, y)$ and $x \cdot y$, respectively. The latter shall be used in case of nested inner products. The unit outward normal and tangential vectors to a domain $\Omega$ shall be denoted by 
$\mathbf{n}=\left(n_{1}, n_{2}\right)$ and $\boldsymbol{\tau}=\left(-n_{2}, n_{1}\right)$, respectively. For a given matrix $A$, we denote by $A^{t}$ its transpose and by $A^{-t}$ the transpose of its inverse.

Function spaces: Denote by $\mathrm{C}_{b}^{k}\left(\mathbb{R}^{2}\right)$ the spaces of $k$-times continuously differentiable scalarvalued functions $u$ with $D^{\beta} u$ bounded whenever $0 \leqslant|\beta| \leqslant k$, where $\beta$ is a multi-index, and equipped with the standard $\mathrm{C}^{k}$-norm. We write $\mathrm{C}_{b}^{k, \alpha}\left(\mathbb{R}^{2}\right), 0<\alpha \leqslant 1$ for the space of functions $u \in \mathrm{C}_{b}^{k}\left(\mathbb{R}^{2}\right)$ such that $D^{\beta} u$ is Hölder continuous with exponent $\alpha$ whenever $|\beta|=k$. The space $\mathrm{C}_{b}^{k, \alpha}\left(\mathbb{R}^{2}\right)$ equipped with the norm

$$
\|u\|_{k, \alpha}:=\sum_{|\beta| \leqslant k} \sup _{\delta}\left|D^{\beta} u\right|+\sum_{|\beta|=k} \sup _{x, y \in \delta, x \neq y} \frac{\left|D^{\beta} u(x)-D^{\beta} u(y)\right|}{|x-y|^{\alpha}}
$$

is a Banach space.

Let $\delta \subset \mathbb{R}^{2}$ be a bounded domain, we also consider the Hölder spaces $\mathrm{C}^{k, \alpha}(\overline{\mathcal{S}})$. We denote by $W^{m, p}(\mathcal{S}), m \in \mathbb{N}, 1 \leqslant p \leqslant \infty$ the standard $L^{p}$-Sobolev space of order $m$ :

$$
W^{m, p}(\delta):=\left\{u \in L^{p}(\delta) \mid D^{\beta} u \in L^{p}(\delta), \text { for } 0 \leqslant|\beta| \leqslant m\right\},
$$

where $D^{\beta}$ is the weak (or distributional) partial derivative. The norm $\|\cdot\|_{W^{m, p}(\delta)}$ associated with $W^{m, p}(\mathcal{S})$ is given by

$$
\|u\|_{W^{m, p}(\mathcal{S})}=\left(\sum_{|\beta| \leqslant m} \int_{\delta}\left|D^{\beta} u\right|^{p} d \mathbf{x}\right)^{1 / p} .
$$

When $p=2$ we write $H^{m}(\delta):=W^{m, 2}(\delta)$ for simplicity. We also denote

$$
H_{\Lambda}^{1}(\delta):=\left\{u \in H^{1}(\delta) \mid u=0 \text { on } \Lambda\right\},
$$

where $\Lambda \subset \partial S$ and $H_{0}^{1}(S)$ when $\Lambda=\partial S$. When the function is vector-valued, we write $\mathrm{C}^{k, \alpha}\left(\overline{\mathcal{S}}, \mathbb{R}^{2}\right), \mathrm{C}_{b}^{k, \alpha}\left(\mathbb{R}^{2}, \mathbb{R}^{2}\right), H^{m}\left(\mathcal{S}, \mathbb{R}^{2}\right)$, and so on for the function spaces.

Domains: The notation $|\Omega|$ denotes the Lebesgue measure of a set $\Omega$ and $\Omega^{c}$ stands for its complement. A domain $\Omega$ is said to be of class $\mathrm{C}^{k}$ or $\mathrm{C}^{k, \alpha}$ if its boundary is locally the graph of a $\mathrm{C}^{k}$ or $\mathrm{e}^{k, \alpha}$ function, respectively; see [8, Ch. 2, Def. 3.1]. We write $\mathbb{1}_{\Omega}$ for the characteristic function of a set $\Omega$, i.e.,

$$
\mathbb{1}_{\Omega}(x)=\left\{\begin{array}{lll}
1 & \text { if } & x \in \Omega \\
0 & \text { if } & x \notin \Omega
\end{array}\right.
$$

For a domain $\Omega$ of class $\mathrm{C}^{2}$ and a vector $\mathbf{v} \in \mathrm{e}^{1}\left(\mathbb{R}^{2}, \mathbb{R}^{2}\right)$, its tangential gradient $\nabla_{\Gamma} \mathbf{v}$ is defined as

$$
\nabla_{\Gamma} \mathbf{v}:=\left.\nabla \mathbf{v}\right|_{\Gamma}-\left(\partial_{n} \mathbf{v}\right) \mathbf{n}
$$

and its tangential divergence $\operatorname{div}_{\Gamma}(\mathbf{v})$ is defined as

$$
\operatorname{div}_{\Gamma}(\mathbf{v}):=\operatorname{div}(\mathbf{v})-D \mathbf{v} \mathbf{n} \cdot \mathbf{n} .
$$

If $\mathbf{v}$ is only defined on $\Gamma$, then the tangential gradient and tangential divergence are defined similarly using an extension of $\mathbf{v}$ to $\mathbb{R}^{2}$ and they are independent of this extension. If the domain has enough regularity, the curvature $\mathcal{H}$ is given by $\mathcal{H}=\operatorname{div}_{\Gamma} \mathbf{n}$. 


\subsection{Existence of solutions for the free boundary problem}

In this paper we work with bounded domains $\omega \in U_{a d}$ where the admissible set of domains is

$$
\begin{aligned}
U_{a d}:=\left\{\omega \subset \mathbb{R}^{2} \mid \omega_{\text {min }} \subset \omega \subset \omega_{\max } \subset \mathcal{E}, \omega\right. & \text { is star-like with respect to all points } \\
& \text { in the ball } \left.B_{\delta}(0) \text { and } \omega \text { is of class } e^{2, \alpha}\right\},
\end{aligned}
$$

where $\omega_{\min }, \omega_{\max }$ are given non-empty domains in $\mathbb{R}^{2}, \omega_{\min }$ contains the origin, $0<\alpha<1$ and the radius $\delta>0$ is a given constant; see [24]. This choice of $u_{a d}$ guarantees existence, uniqueness as well as stability (in the sense of [1, Theorem 3.9]) of the solution to $\left(\mathcal{F}_{\omega}\right)$ with respect to $\omega$. Moreover, it is shown in [1] that if $\omega \in \mathcal{U}_{a d}$, then the boundary $\partial \Omega^{*}(\omega)$ is of class $\mathrm{C}^{\infty}$ and is star-like with respect to all points in $B_{\delta}(0)$. Here $\Omega^{*}(\omega)$ denotes the solution to $\left(\mathcal{F}_{\omega}\right)$.

\subsection{Fixed point approach for the free boundary problem}

The free boundary problem $\left(\mathcal{F}_{\omega}\right)$ can be formulated as a shape optimization problem [10, 14]. In this way, the numerical solution of $\left(\mathcal{F}_{\omega}\right)$ relies on the use of gradient information that depends on known state and adjoint systems. On the other hand, it would be helpful to have a method that solves $\left(\mathfrak{F}_{\omega}\right)$ using some simple updating formula based only on the solution of some state system. The structure of such a scheme is as follows:

(1) Choose an initial approximation of the free boundary.

(2) Solve the boundary value problem (1.1)-(1.4) for $u$ with one condition on $\Gamma$ omitted.

(3) Update $\Gamma$ using the discrepancy left by the remaining boundary condition.

(4) Iterate from step (2) until stationarity up to a specified accuracy is reached.

This scheme is simple to implement but it is not obvious how to construct the updating step in such a manner that the method converges and that the convergence is fast. In order to obtain an optimal updating step, Tilhonen [23] derived the first and second order derivatives for the cost $g(\Omega)$ in the following shape optimization problem:

$$
\begin{array}{ll}
\text { minimize } & \mathcal{l}(\Omega):=\frac{1}{2} \int_{\Gamma} u_{\Omega}^{2} d s \\
\text { subject to } & \Omega \in \mathcal{O}_{a d}, u_{\Omega} \in H^{1}(\Omega \backslash \bar{\omega})
\end{array}
$$

with

$$
\begin{aligned}
-\Delta u_{\Omega}=0 & \text { in } \quad \Omega \backslash \bar{\omega}, \\
u_{\Omega}=1 & \text { on } \quad \Sigma, \\
\alpha u_{\Omega}+\partial_{n} u_{\Omega}=\mu & \text { on } \quad \Gamma .
\end{aligned}
$$

Here the coefficient $\alpha$ can be chosen freely without affecting the solution of the free boundary problem provided that the solution to (2.5) is such that $\left.u_{\Omega}\right|_{\Gamma}=0$. However, changes in $\alpha$ affect the conditioning of the Hessian of the cost functional $\mathcal{l}(\Omega)$. It has been shown in [23] that $\alpha=$ $\mathcal{H}$, where $\mathcal{H} \geqslant 0$ is the mean curvature of $\Gamma$, is the optimal choice for an efficient resolution of the optimization problem (2.5). Furthermore, it has been shown in [9], using formal asymptotic expansions, that the optimal updating step may be approximated by

$$
\mathbf{x}^{(k+1)}=\mathbf{x}^{(k)}-\frac{u\left(\mathbf{x}^{(k)}\right)}{\mu} \mathbf{n}_{\varepsilon}\left(\mathbf{x}^{(k)}\right),
$$


where $\mathbf{x}^{(k)}:=\left(x_{1}^{(k)}, x_{2}^{(k)}\right) \in \Gamma^{(k)}$ is the $k$-th iterate and $\mathbf{n}_{\varepsilon} \in H^{1}\left(\Gamma, \mathbb{R}^{2}\right)$ is the smoothed normal vector field on the free boundary $\Gamma$ satisfying

$$
\int_{\Gamma} \varepsilon \nabla_{\Gamma} \mathbf{n}_{\varepsilon}: \nabla_{\Gamma} \boldsymbol{v}+\mathbf{n}_{\varepsilon} \cdot \boldsymbol{v} d s=\int_{\Gamma} \mathbf{n} \cdot \boldsymbol{v} d s \text { for all } \boldsymbol{v} \in H^{1}\left(\Gamma, \mathbb{R}^{2}\right),
$$

and $\varepsilon$ is some fixed small parameter. The mean curvature $\mathcal{H}$ of $\Gamma$ is defined as

$$
\mathcal{H}:=\operatorname{div}_{\Gamma}\left(\mathbf{n}_{\varepsilon}\right)
$$

The algorithm to update the free boundary $\Gamma$ at the $k^{t h}$ step now becomes

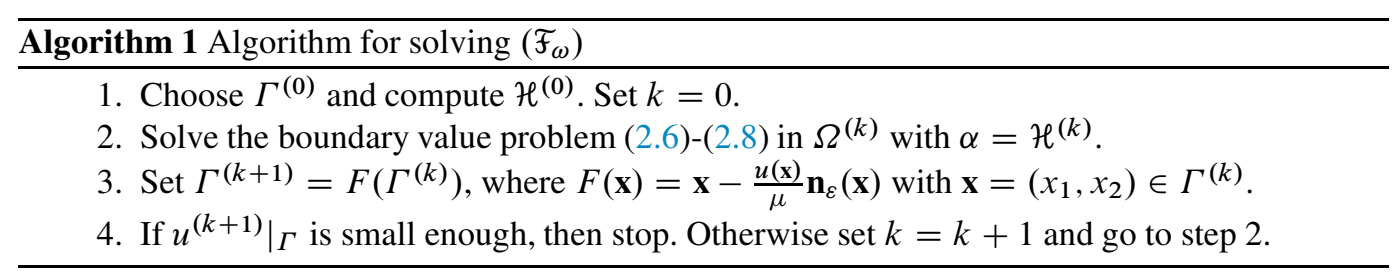

Flucher and Rumpf [9] analyzed the convergence of Algorithm 1 in the continuous case. Their analysis shows that the convergence suffers from the smoothing procedure so that the convergence is less than quadratic but still super-linear. In two dimensions, one can obtain the convergence rate of order $3 / 2$.

\subsection{Bilevel shape optimization problems}

Under the assumptions in Section 2.2, the solution of $\left(\mathcal{F}_{\omega}\right)$ is unique. Thus there exists a mapping

$$
\Omega^{*}: U_{a d} \ni \omega \mapsto \Omega^{*}(\omega) \in \mathcal{O}_{a d},
$$

such that $\Omega^{*}(\omega)$ is the solution of $\left(\mathcal{F}_{\omega}\right)$. We denote $\Gamma^{*}(\omega):=\partial \Omega^{*}(\omega)$.

We next turn to a shape optimization problem with respect to $\omega$. Our control objective consists in determining $\omega$ such that $\Gamma^{*}(\omega)$ is as close as possible to the boundary $\partial E$ of a target Lipschitz domain $E \in \mathcal{O}_{a d}$ such that $\omega \subset E$.

We study two functionals which allow us to achieve this goal. The first one is

$$
J_{1}(\Omega):=\left|\Omega \cap E^{c}\right|+\left|E \cap \Omega^{c}\right| .
$$

The term $\left|\Omega \cap E^{c}\right|=0$ forces $\Omega$ to be included in $E$ while $\left|E \cap \Omega^{c}\right|=0$ forces $\Omega$ to contain $E$.

We may also write $J_{1}$ as

$$
J_{1}(\Omega)=\int_{\Omega \cap E^{c}} 1 d x+\int_{E \cap \Omega^{c}} 1 d x
$$

Another approach consists in minimizing the functional

$$
J_{2}(\Omega, \omega)=\frac{1}{2} \int_{\Omega \cap E^{c}} u(\Omega, \omega)^{2} d x+\frac{1}{2} \int_{E \cap \omega^{c}}\left(u(\Omega, \omega)-u_{l}(\omega)\right)^{2} d x,
$$




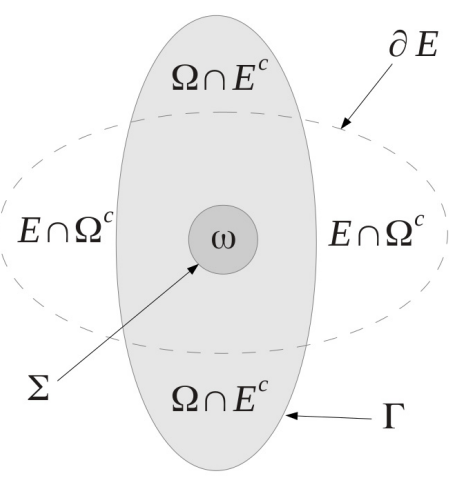

FIG. 2. "Free" set $\Omega$ and target $E$

where $u=u(\Omega, \omega) \in H_{0}^{1}(\mathcal{E})$ is the extension by zero to $\varepsilon$ of the solution of

$$
\begin{array}{rlll}
-\Delta u & =0 & \text { in } & \Omega \backslash \bar{\omega}, \\
u=1 & \text { on } & \Sigma:=\partial \omega, \\
u=0 & \text { on } & \Gamma:=\partial \Omega .
\end{array}
$$

Such an extension exists as soon as $\Omega \subset \mathcal{E}$ is measurable and $u=0$ on $\partial \Omega$; see [13]. Note that $u\left(\Omega^{*}(\omega), \omega\right)$ solves (1.1)-(1.4). The function $u_{l}=u_{l}(\omega)$ solves the linear problem

$$
\begin{aligned}
-\Delta u_{l}=0 & \text { in } E \backslash \bar{\omega}, \\
u_{l}=1 & \text { on } \Sigma, \\
u_{l}=0 & \text { on } \partial E,
\end{aligned}
$$

and is also extended by zero to a function in $H_{0}^{1}(\varepsilon)$. The following proposition shows that minimizing $J_{1}$ and $J_{2}$ allows to drive $\Omega$ to $E$.

Proposition 2.1 Let $\omega$ be a given open bounded set with $\omega \subset \Omega$. We have $J_{1}(\Omega)=0$ and $J_{2}(\Omega, \omega)=0$ if and only if $\Omega=E$ almost everywhere.

Proof. We start with the case of $J_{1}$. If $\Omega=E$, then obviously $J_{1}(E)=0$. On the other hand, if $J_{1}(\Omega)=0$, then $\left|\Omega \cap E^{c}\right|=\left|E \cap \Omega^{c}\right|=0$ and thus $\Omega=E$ almost everywhere.

Now we consider the case of $J_{2}$. Observe that if $\Omega=E$, then $\Omega \cap E^{c}=\emptyset, u=u_{l}$ a.e on $E \cap \omega^{c}$ and thus $J_{2}(\Omega, \omega)=0$. Conversely, we show that if $J_{2}(\Omega, \omega)=0$, then $\Omega=E$ almost everywhere. Since $\Omega=E$ if and only if $\left|\Omega \cap E^{c}\right|+\left|\Omega^{c} \cap E\right|=0$, it suffices to show that $J_{2}(\Omega, \omega)=0$ implies that $\left|\Omega \cap E^{c}\right|+\left|\Omega^{c} \cap E\right|=0$. We use a contradiction argument to support the latter assertion.

To this end, suppose that $\left|\Omega \cap E^{c}\right|+\left|\Omega^{c} \cap E\right| \neq 0$ and $J_{2}(\Omega, \omega)=0$. Then we face two possibilities: (i) $\left|\Omega^{c} \cap E\right|>0$ or (ii) $\left|\Omega^{c} \cap E\right|=0$ and $\left|\Omega \cap E^{c}\right|>0$. In case (i), since $J_{2}(\Omega, \omega)=$ 0 , we have $u-u_{l}=0$ almost everywhere on $E \cap \omega^{c}$. Since $\Omega^{c} \cap E \subset \omega^{c} \cap E$ due to $\bar{\omega} \subset \Omega$ we have $u_{l}=u=0$ on $\Omega^{c} \cap E$ which is a contradiction since $u_{l}>0$ in $\Omega^{c} \cap E$ due to the maximum principle. Hence case (i) cannot happen. 
We turn to case (ii). Since $J_{2}(\Omega, \omega)=0$ and $\left|\Omega \cap E^{c}\right|>0$ we have $u=0$ almost everywhere on $\Omega \cap E^{c}$ which is a contradiction since $u>0$ in $\Omega \cap E^{c}$ due to the maximum principle.

Hence we conclude that $\Omega=E$ almost everywhere whenever $J_{2}(\Omega, \omega)=0$.

Writing $J_{1}(\Omega, \omega)=J_{1}(\Omega)$ for generality, we can now formulate the bilevel shape optimization problem as

$$
\left(\mathbb{B}_{i}\right): \begin{cases}\text { minimize } & J_{i}(\Omega, \omega) \\ \text { subject to } & \omega \in \mathcal{U}_{a d} \text { and } \Omega \text { solves }\left(\mathcal{F}_{\omega}\right) .\end{cases}
$$

The problem of minimizing $J_{i}(\Omega, \omega)$ over $\omega \in U_{a d}$ is called the upper-level problem, while the problem of solving $\left(\mathcal{F}_{\omega}\right)$ is called the lower-level problem. Similarly, $\Omega$ is the lower-level variable while $\omega$ is the upper-level variable. Defining the associated functionals

$$
\begin{aligned}
& K_{1}(\omega):=J_{1}\left(\Omega^{*}(\omega)\right) \\
& K_{2}(\omega):=J_{2}\left(\Omega^{*}(\omega), \omega\right),
\end{aligned}
$$

we can rewrite the bilevel problem as

$$
\left(B_{i}\right): \begin{cases}\text { minimize } & K_{i}(\omega) \\ \text { subject to } & \omega \in \mathcal{U}_{a d} .\end{cases}
$$

REMARK 2.2 Note that the minimum of $K_{1}(\omega)$ and $K_{2}(\omega)$ need not exist and need not be 0 in general. In these cases we have $\Omega^{*}(\omega) \neq E$ even if $\omega$ minimizes $K_{i}(\omega)$. Indeed we have $K_{i}(\omega)=$ $J_{i}\left(\Omega^{*}(\omega), \omega\right)$ but $\Omega^{*}\left(\mathcal{U}_{a d}\right) \subsetneq \mathcal{O}_{a d}$ in general. So if $E \in \mathcal{O}_{a d} \backslash \Omega^{*}\left(\mathcal{U}_{a d}\right)$, then we cannot find $\omega$ such that $K_{i}(\omega)=0$. It is easily seen that $\Omega^{*}\left(U_{a d}\right) \neq \mathcal{O}_{a d}$ in general since the domains $\Omega^{*}(\omega)$ have $\mathrm{C}^{\infty}$ regularity due to our choice of $U_{a d}$ (see Section 2.2). However, if $\omega$ minimizes $K_{i}(\omega)$, then $\Omega^{*}(\omega)$ is the closest approximation of $E$ (for $K_{i}(\omega)$ ) which solves the free boundary problem $\left(\mathcal{F}_{\omega}\right)$. We observe this phenomenon in Section 4.1 .3 of the numerical results.

\section{Sensitivity analysis}

\subsection{Perturbation of identity}

In the study of the optimization problem $\left(\mathbb{B}_{i}\right)$, several issues arise including the sensitivity of $\Omega^{*}(\omega)$ with respect to $\omega$. To deal with these issues, concepts of shape differential calculus, described in detail in the monographs $[8,13,18,22]$, are utilized. The inherent difficulty in dealing with shape functionals lies in the fact that sets of shapes are not vector spaces and the notion of differentiation cannot be used directly. Instead, one may consider perturbations of a reference shape by means of transformations in an appropriate function space which allows differentiation of the functional. These transformations can be constructed, for instance, by perturbation of the identity [8] or by the flow of a velocity field $[8,22]$. We will use the perturbation of identity method in what follows. To this end let $\mathbf{V} \in \mathrm{C}_{b}^{k, \alpha}\left(\mathbb{R}^{2}, \mathbb{R}^{2}\right)$ with $k \geqslant 1$ and $0<\alpha<1$. We consider perturbations of identity $I+\mathbf{V}$ where $\mathbf{V}$ is in a neighborhood of 0 in $\mathrm{C}_{b}^{k, \alpha}\left(\mathbb{R}^{2}, \mathbb{R}^{2}\right)$ so that $I+\mathbf{V}$ is a bi-Lipschitz homeomorphism. In what follows we will denote by

$$
\delta_{\mathbf{V}}:=(I+\mathbf{V})(\delta)
$$


the transformation of a generic domain $\delta$ by $I+\mathbf{V}$. Let $K(\omega)$ be a real-valued functional associated with $\omega \subset \mathbb{R}^{2}$. The functional $K(\omega)$ is Fréchet-differentiable at $\omega$ if there exists a linear and continuous functional $\nabla K(\omega)$ from $\mathrm{e}_{b}^{k, \alpha}\left(\mathbb{R}^{2}, \mathbb{R}^{2}\right)$ to $\mathbb{R}$ called shape gradient such that

$$
K\left(\omega_{\mathbf{V}}\right)=K(\omega)+\nabla K(\omega) \cdot \mathbf{V}+r(\mathbf{V})
$$

where $|r(\mathbf{V})| /\|\mathbf{V}\|_{k, \alpha} \rightarrow 0$ as $\|\mathbf{V}\|_{k, \alpha} \rightarrow 0$. In this case one defines the shape derivative as

$$
d K(\omega ; \mathbf{V}):=\nabla K(\omega) \cdot \mathbf{V}
$$

We have that

$$
\mathrm{C}_{b}^{k, \alpha}\left(\mathbb{R}^{2}, \mathbb{R}^{2}\right) \ni \mathbf{V} \mapsto d K(\omega ; \mathbf{V})
$$

is a distribution on $\mathbb{R}^{2}$ with support on $\Sigma=\partial \omega$. In addition, if $\omega$ is of class $\mathbb{C}^{k+1, \alpha}$, then for all $\mathbf{V} \in \mathbb{C}_{b}^{k, \alpha}\left(\mathbb{R}^{2}, \mathbb{R}^{2}\right)$ such that $\mathbf{V} \cdot \mathbf{n}=0$ on $\Sigma$, we have $d K(\omega ; \mathbf{V})=0$. In other words, the shape derivative in direction $\mathbf{V}$ depends only on the normal component of the trace of $\mathbf{V}$ on $\Sigma$. This is the so-called Hadamard-Zolésio structure theorem in, e.g., [8, Ch. 8] or [13, Ch. 5]. If we assume that the data is smooth enough, then there exists an integrable function $g$ such that the shape derivative can be expressed as

$$
d K(\omega ; \mathbf{V})=\int_{\Sigma} g \mathbf{V} \cdot \mathbf{n} d s .
$$

A similar definition can be used for the shape derivative of functionals taking their values in a Banach space. In particular, we would like to define the shape derivative of the solution of a partial differential equation such as (1.1)-(1.4). Let $u_{\mathbf{V}}$ denote the solution of a partial differential equation on the perturbed domain $\omega_{\mathbf{V}}$. Since $u_{\mathbf{V}}$ lives in a function space which depends on the moving domain $\omega_{\mathbf{V}}$, one cannot compute the shape derivative directly. Instead we take the derivative of $u_{\mathbf{V}} \circ(I+\mathbf{V})$, which is defined on $\omega$, with respect to $\mathbf{V}$ in a direction $\widehat{\mathbf{V}}$; the latter is called material derivative and written $\dot{u}(\mathbf{V} ; \widehat{\mathbf{V}})$. Then one introduces the shape derivative by means of:

$$
u^{\prime}(\mathbf{V} ; \widehat{\mathbf{V}}):=\dot{u}(\mathbf{V} ; \widehat{\mathbf{V}})-\nabla u \cdot \widehat{\mathbf{V}} .
$$

Since one usually considers $u^{\prime}(0 ; \widehat{\mathbf{V}})$ the notation $u^{\prime}(\widehat{\mathbf{V}}):=u^{\prime}(0 ; \widehat{\mathbf{V}})$ is used for simplicity.

\subsection{Sensitivity of $u$ with respect to $\omega$}

In order to compute (3.1), we proceed by first computing formally the derivative of the solution to (1.1)-(1.4) with respect to $\omega$, using the classical results of shape calculus; see for instance [22, Section 3]. To this end, let $T(\mathbf{V})=I+\mathbf{V}$ be the transformation associated with a vector field $\mathbf{V} \in \mathrm{C}_{b}^{k, \alpha}\left(\mathbb{R}^{2}, \mathbb{R}^{2}\right)$, and denote

$$
\omega_{\mathbf{V}}=T(\mathbf{V})(\omega)
$$

Assume that there exists $\mathbf{W}^{*} \in \mathbb{C}_{b}^{k, \alpha}\left(\mathbb{R}^{2}, \mathbb{R}^{2}\right)$ such that $\Omega^{*}\left(\omega_{\mathbf{V}}\right)=T\left(\mathbf{W}^{*}\right)\left(\Omega^{*}\left(\omega_{0}\right)\right)$ for $\mathbf{V}$ in a neighborhood of 0 , where $\Omega^{*}\left(\omega_{\mathbf{V}}\right)$ is the solution to $\left(\mathcal{F}_{\omega_{\mathbf{V}}}\right)$ and $\omega_{0}=\omega$. If such a $\mathbf{W}^{*}$ exists, then it depends on $\mathbf{V}$. In Theorem 3.3 we prove the existence of $\mathbf{W}^{*}$ as a function of $\mathbf{V}$. Here we formally compute the first-order approximation of $\mathbf{W}^{*}$ with respect to $\mathbf{V}$. To obtain such a result, we study the sensitivity of the solution $u$ to (1.1)-(1.4) with respect to $\omega$. According to [22, pp. 118-120], the 
shape derivative $u^{\prime}\left(\widehat{\mathbf{V}}, \widehat{\mathbf{W}}^{*}\right)$ of $u$ solution of (1.1)-(1.4) with respect to both transformations $T(\mathbf{V})$ and $T\left(\mathbf{W}^{*}\right)$ at $\mathbf{V}=0$ and $\mathbf{W}^{*}=0$ in directions $\widehat{\mathbf{V}}$ and $\widehat{\mathbf{W}}^{*}$ satisfies

$$
\begin{aligned}
-\Delta u^{\prime}\left(\widehat{\mathbf{V}}, \widehat{\mathbf{W}}^{*}\right) & =0 & & \text { in } \Omega^{*}(\omega) \backslash \bar{\omega}, \\
u^{\prime}\left(\widehat{\mathbf{V}}, \widehat{\mathbf{W}}^{*}\right) & =-\partial_{n} u \widehat{\mathbf{V}} \cdot \mathbf{n} & & \text { on } \Sigma, \\
u^{\prime}\left(\widehat{\mathbf{V}}, \widehat{\mathbf{W}}^{*}\right) & =-\partial_{n} u \widehat{\mathbf{W}}^{*} \cdot \mathbf{n} & & \text { on } \Gamma^{*}(\omega), \\
\partial_{n} u^{\prime}\left(\widehat{\mathbf{V}}, \widehat{\mathbf{W}}^{*}\right) & =\operatorname{div}_{\Gamma}\left(\nabla_{\Gamma} u \widehat{\mathbf{W}}^{*} \cdot \mathbf{n}\right)+\mu \nVdash \widehat{\mathbf{W}}^{*} \cdot \mathbf{n} & & \text { on } \Gamma^{*}(\omega),
\end{aligned}
$$

where $\widehat{\mathbf{V}}$ and $\widehat{\mathbf{W}}^{*}$ are chosen such that they have compact supports in neighborhoods of $\Sigma$ and $\Gamma^{*}(\omega)$, respectively, i.e., $\widehat{\mathbf{V}}=0$ on $\Gamma^{*}(\omega)$ and $\widehat{\mathbf{W}}^{*}=0$ on $\Sigma$. In view of (1.1)-(1.4) we have $\nabla_{\Gamma} u=0$ and $\partial_{n} u=\mu$ on $\Gamma$, and we may simplify (3.5)-(3.8) as

$$
\begin{aligned}
-\Delta u^{\prime}\left(\widehat{\mathbf{V}}, \widehat{\mathbf{W}}^{*}\right) & =0 & & \text { in } \Omega^{*}(\omega) \backslash \bar{\omega}, \\
u^{\prime}\left(\widehat{\mathbf{V}}, \widehat{\mathbf{W}}^{*}\right) & =-\partial_{n} u \widehat{\mathbf{V}} \cdot \mathbf{n} & & \text { on } \Sigma, \\
u^{\prime}\left(\widehat{\mathbf{V}}, \widehat{\mathbf{W}}^{*}\right) & =-\mu \widehat{\mathbf{W}}^{*} \cdot \mathbf{n} & & \text { on } \Gamma^{*}(\omega), \\
\partial_{n} u^{\prime}\left(\widehat{\mathbf{V}}, \widehat{\mathbf{W}}^{*}\right) & =\mu \psi \widehat{\mathbf{W}}^{*} \cdot \mathbf{n} & & \text { on } \Gamma^{*}(\omega) .
\end{aligned}
$$

Since $\widehat{\mathbf{W}}^{*}=\widehat{\mathbf{W}}^{*}(\widehat{\mathbf{V}})$, we actually have $u^{\prime}\left(\widehat{\mathbf{V}}, \widehat{\mathbf{W}}^{*}\right)=u^{\prime}(\widehat{\mathbf{V}})$. Indeed, gathering the boundary conditions (3.11) and (3.12), we obtain the following partial differential equation with Robin boundary conditions on $\Gamma=\Gamma^{*}(\mathbf{W})$ for $u^{\prime}(\mathbf{V})$

$$
\begin{aligned}
-\Delta u^{\prime}(\widehat{\mathbf{V}}) & =0 & & \text { in } \Omega^{*}(\omega) \backslash \bar{\omega}, \\
u^{\prime}(\widehat{\mathbf{V}}) & =-\partial_{n} u \widehat{\mathbf{V}} \cdot \mathbf{n} & & \text { on } \Sigma, \\
\partial_{n} u^{\prime}(\widehat{\mathbf{V}})+\mathcal{H} u^{\prime}(\widehat{\mathbf{V}}) & =0 & & \text { on } \Gamma^{*}(\omega) .
\end{aligned}
$$

Assuming $\mathcal{H} \geqslant 0$, equation (3.13)-(3.15) has a unique solution $u^{\prime}(\widehat{\mathbf{V}})$; see Lemma 3.1. Using boundary conditions (3.11), we formally obtain

$$
\widehat{\mathbf{W}}^{*}(\widehat{\mathbf{V}})=-\mu^{-1} u^{\prime}(\widehat{\mathbf{V}}) \mathbf{n} \quad \text { on } \Gamma^{*}(\omega)
$$

and the normal component of $\widehat{\mathbf{W}}^{*}(\widehat{\mathbf{V}})$ is uniquely defined on $\Gamma$. The tangential component of $\widehat{\mathbf{W}}^{*}$ can be chosen arbitrarily according to the Hadamard structure theorem [22, p. 59] mentioned in Section 3.1 and we take it equal to zero.

In fact, since $\mathbf{W}^{*}=\mathbf{W}^{*}(\mathbf{V})$, we have $\widehat{\mathbf{W}}^{*}(\widehat{\mathbf{V}})=D_{\mathbf{V}} \mathbf{W}^{*}(0 ; \widehat{\mathbf{V}})$ which is a first order approximation of $\mathbf{W}^{*}(\widehat{\mathbf{V}})$. We now show the existence of $\mathbf{W}^{*}(\mathbf{V})$ in Theorem 3.3, for which we first require the following preliminary lemmata. Further we prove formula (3.16) in Corollary 3.4.

LEMMA 3.1 Let $m \geqslant 2$ be an integer and $0<\alpha<1$. If $\psi \in \mathrm{C}^{m-1, \alpha}(\Gamma), \Omega$ is bounded of class $\mathrm{e}^{m+1, \alpha}, \omega$ is bounded of class $\mathrm{C}^{m, \alpha}$ and $\mathcal{H} \geqslant 0$ on $\Gamma$, then the linearized system

$$
\begin{aligned}
-\Delta v=0 & & \text { in } \Omega \backslash \bar{\omega}, \\
v=0 & & \text { on } \Sigma, \\
\partial_{n} v+\mathcal{H} v=\psi & & \text { on } \Gamma,
\end{aligned}
$$

admits a unique solution $v \in \mathrm{e}^{m, \alpha}(\bar{\Omega} \backslash \omega)$. 
Proof. If $\Omega$ is of class $\mathrm{e}^{m+1, \alpha}$, then $\mathcal{H}=\operatorname{div}_{\Gamma}(\mathbf{n})$ is of class $\mathrm{C}^{m-1, \alpha}$. Applying standards regularity results for elliptic operators we obtain the result; see [26, Lemma 3.19].

We introduce the functions $u_{1, \mathbf{v}, \mathbf{w}}$ and $u_{2, \mathbf{v}, \mathbf{w}}$, solutions of

$$
\begin{aligned}
-\Delta u_{1, \mathbf{v}, \mathbf{W}}=0 & \text { in } \Omega_{\mathbf{V}+\mathbf{W}} \backslash \overline{\omega_{\mathbf{V}+\mathbf{W}}} \\
u_{1, \mathbf{V}, \mathbf{W}}=1 & \text { on } \Sigma_{\mathbf{V}+\mathbf{W}}, \\
u_{1, \mathbf{v}, \mathbf{W}}=0 & \text { on } \Gamma_{\mathbf{V}+\mathbf{W}},
\end{aligned}
$$

and

$$
\begin{aligned}
-\Delta u_{2, \mathbf{V}, \mathbf{W}}=0 & \text { in } \Omega_{\mathbf{V}+\mathbf{W}} \backslash \overline{\omega_{\mathbf{V}+\mathbf{W}}} \\
u_{2, \mathbf{V}, \mathbf{W}}=1 & \text { on } \Sigma_{\mathbf{V}+\mathbf{W}}, \\
\partial_{n} u_{2, \mathbf{v}, \mathbf{W}}=\mu & \text { on } \Gamma_{\mathbf{V}+\mathbf{W}},
\end{aligned}
$$

respectively. It is convenient to introduce the notation

$$
\mathfrak{C}:=\mathrm{C}_{b}^{m, \alpha}\left(\mathbb{R}^{2}, \mathbb{R}^{2}\right) \times \mathrm{C}_{b}^{m, \alpha}\left(\mathbb{R}^{2}, \mathbb{R}^{2}\right) .
$$

LEMMA 3.2 Let $m \geqslant 2,0<\alpha<1, \Omega, \omega$ be bounded of class $\mathrm{e}^{m, \alpha}$ and $(\mathbf{V}, \mathbf{W}) \in \mathfrak{C}$. Then the functions

$$
\mathfrak{C} \ni(\mathbf{V}, \mathbf{W}) \rightarrow u_{1, \mathbf{V}, \mathbf{W}} \circ(I+\mathbf{V}+\mathbf{W}) \in \mathrm{C}^{m, \alpha}(\bar{\Omega} \backslash \omega)
$$

and

$$
\mathfrak{C} \ni(\mathbf{V}, \mathbf{W}) \rightarrow u_{2, \mathbf{V}, \mathbf{W}} \circ(I+\mathbf{V}+\mathbf{W}) \in \mathrm{C}^{m, \alpha}(\bar{\Omega} \backslash \omega)
$$

are of class $\mathrm{C}^{\infty}$ in a neighborhood of $(0,0) \in \mathfrak{C}$.

Proof. We only prove the case of $u_{2, \mathrm{~V}, \mathrm{~W}}$, the case of $u_{1, \mathrm{~V}, \mathrm{~W}}$ being a straightforward adaptation of

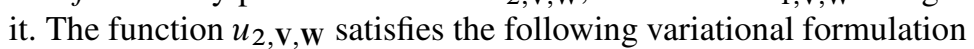

$$
\int_{\Omega_{\mathbf{V}+\mathbf{W}} \backslash \overline{\omega_{\mathbf{V}+\mathbf{W}}}} \nabla u_{2, \mathbf{V}, \mathbf{W}} \cdot \nabla \hat{\varphi}=\mu \int_{\Gamma_{\mathbf{V}+\mathbf{W}}} \hat{\varphi} \quad \text { for all } \hat{\varphi} \in H_{\Sigma_{\mathbf{V}+\mathbf{W}}}^{1}\left(\Omega_{\mathbf{V}+\mathbf{W}} \backslash \overline{\omega_{\mathbf{V}+\mathbf{W}}}\right) .
$$

Transporting back the problem on $\Omega \backslash \bar{\omega}$ by using the transformation $(I+\mathbf{V}+\mathbf{W})^{-1}$ we obtain

$$
\int_{\Omega \backslash \bar{\omega}} A(\mathbf{V}, \mathbf{W}) \nabla z_{\mathbf{V}, \mathbf{W}} \cdot \nabla \varphi=\mu \int_{\Gamma} \varphi J_{\Gamma, \mathbf{V}, \mathbf{W}} \quad \text { for all } \varphi \in H_{\Sigma}^{1}(\Omega \backslash \bar{\omega}),
$$

with

$$
\begin{aligned}
z_{\mathbf{V}, \mathbf{W}} & :=u_{2, \mathbf{V}, \mathbf{W}} \circ(I+\mathbf{V}+\mathbf{W}), \\
\varphi & :=\hat{\varphi} \circ(I+\mathbf{V}+\mathbf{W}), \\
A(\mathbf{V}, \mathbf{W}) & :=J_{\mathbf{V}, \mathbf{W}}(I+D \mathbf{V}+D \mathbf{W})^{-1}\left(I+D \mathbf{V}^{t}+D \mathbf{W}^{t}\right)^{-1}, \\
J_{\mathbf{V}, \mathbf{W}} & :=\operatorname{det}(I+D \mathbf{V}+D \mathbf{W}), \\
J_{\Gamma, \mathbf{V}, \mathbf{W}} & :=\operatorname{det}(I+D \mathbf{V}+D \mathbf{W})\left\|(I+D \mathbf{V}+D \mathbf{W})^{-t} \mathbf{n}\right\|,
\end{aligned}
$$


where $J_{\mathbf{V}, \mathbf{W}}$ is the Jacobian of transformation $I+\mathbf{V}+\mathbf{W}$ while $J_{\Gamma, \mathbf{V}, \mathbf{w}}$ is the boundary Jacobian on $\Gamma$. The strong form of (3.24) is given by

$$
\begin{aligned}
-\operatorname{div}\left(A(\mathbf{V}, \mathbf{W}) \nabla z_{\mathbf{V}, \mathbf{W}}\right) & =0 & & \text { in } \Omega \backslash \bar{\omega}, \\
z_{\mathbf{V}, \mathbf{W}} & =1 & & \text { on } \Sigma, \\
\left(A(\mathbf{V}, \mathbf{W}) \nabla z_{\mathbf{V}, \mathbf{W}}\right) \cdot \mathbf{n} & =\mu J_{\Gamma, \mathbf{V}, \mathbf{W}} & & \text { on } \Gamma .
\end{aligned}
$$

We introduce the function

$$
\begin{aligned}
G: \mathfrak{C} \times \mathbb{C}^{m, \alpha}(\bar{\Omega} \backslash \omega) & \rightarrow \mathrm{C}^{m-2, \alpha}(\bar{\Omega} \backslash \omega) \times \mathrm{C}^{m-1, \alpha}(\Gamma) \times \mathrm{C}^{m, \alpha}(\Sigma) \\
(\mathbf{V}, \mathbf{W}, z) & \mapsto\left(-\operatorname{div}(A(\mathbf{V}, \mathbf{W}) \nabla z),\left.\left((A(\mathbf{V}, \mathbf{W}) \nabla z) \cdot \mathbf{n}-\mu J_{\Gamma, \mathbf{V}, \mathbf{W}}\right)\right|_{\Gamma},\left.(z-1)\right|_{\Sigma}\right)
\end{aligned}
$$

First of all, the function

$$
\mathfrak{C} \ni(\mathbf{V}, \mathbf{W}) \mapsto J_{\mathbf{V}, \mathbf{W}}=\operatorname{det}(I+D \mathbf{V}+D \mathbf{W}) \in \mathrm{C}_{b}^{m-1, \alpha}\left(\mathbb{R}^{2}, \mathbb{R}\right)
$$

is of class $\mathrm{C}^{\infty}$ since $I+D \mathbf{V}+D \mathbf{W}$ is linear in $(\mathbf{V}, \mathbf{W})$ and the determinant is polynomial and continuous for the $\mathrm{e}^{m, \alpha}$-norm. Writing $(I+D \mathbf{V}+D \mathbf{W})^{-1}=\sum_{q \geqslant 0}(-1)^{q}(D \mathbf{V}+D \mathbf{W})^{q}$, we can see that the function

$$
\mathfrak{C} \ni(\mathbf{V}, \mathbf{W}) \mapsto(I+D \mathbf{V}+D \mathbf{W})^{-1} \in \mathbb{C}_{b}^{m-1, \alpha}\left(\mathbb{R}^{2}, m_{2}\right)
$$

is of class $\mathrm{e}^{\infty}$ for small $(\mathbf{V}, \mathbf{W})$, where $m_{2}$ is the set of $2 \times 2$-matrices. Thus $(\mathbf{V}, \mathbf{W}) \rightarrow A(\mathbf{V}, \mathbf{W})$ is $\mathrm{C}^{\infty}$ for small $(\mathbf{V}, \mathbf{W})$ since the function

$$
\begin{aligned}
\mathrm{C}_{b}^{m-1, \alpha}\left(\mathbb{R}^{2}, m_{2}\right) \times \mathrm{e}^{m, \alpha}(\bar{\Omega} \backslash \omega) & \rightarrow \mathrm{e}^{m-2, \alpha}(\bar{\Omega} \backslash \omega) \times \mathrm{e}^{m-1, \alpha}(\Gamma) \times \mathrm{e}^{m, \alpha}(\Sigma) \\
(A, z) & \mapsto\left(-\operatorname{div}(A \nabla z),\left.((A \nabla z) \cdot \mathbf{n})\right|_{\Gamma},\left.z\right|_{\Sigma}\right)
\end{aligned}
$$

is bilinear and continuous. For small $(\mathbf{V}, \mathbf{W})$ the function $(\mathbf{V}, \mathbf{W}) \mapsto J_{\Gamma, \mathbf{v}, \mathbf{W}}$ is also $\mathrm{e}^{\infty}$. Gathering the previous results we get that $G$ is $\mathrm{C}^{\infty}$. In view of $A(0,0)=I$ and $J_{\Gamma, 0,0}=I$, we compute

$$
D_{z} G\left(0,0, u_{2,0,0} ; \hat{z}\right)=\left(-\Delta \hat{z},\left.\partial_{n} \hat{z}\right|_{\Gamma},\left.z\right|_{\Sigma}\right) .
$$

Since $\Omega$ and $\omega$ are of class $\mathrm{C}^{m, \alpha}, 0<\alpha<1$, regularity theory of elliptic partial differential equations implies that

$$
D_{z} G\left(0,0, u_{2,0,0}\right): \mathrm{C}^{m, \alpha}(\bar{\Omega} \backslash \omega) \rightarrow \mathrm{e}^{m-2, \alpha}(\bar{\Omega} \backslash \omega) \times \mathrm{C}^{m-1, \alpha}(\Gamma) \times \mathrm{C}^{m, \alpha}(\Sigma)
$$

is an isomorphism for $m \geqslant 2$ and $0<\alpha<1$. Therefore, we can apply the implicit function theorem and there exists a function

$$
(\mathbf{V}, \mathbf{W}) \in \mathfrak{C} \mapsto \tilde{z}(\mathbf{V}, \mathbf{W}) \in \mathbb{C}^{m, \alpha}(\bar{\Omega} \backslash \omega)
$$

of class $\mathrm{C}^{\infty}$ on a neighborhood of $(0,0) \in \mathfrak{C}$ such that $G(\mathbf{V}, \mathbf{W}, \tilde{z}(\mathbf{V}, \mathbf{W})) \equiv 0$. By uniqueness of the solution to (3.25)-(3.27), we get $\tilde{z}(\mathbf{V}, \mathbf{W})=z(\mathbf{V}, \mathbf{W})=u_{2, \mathbf{V}, \mathbf{W}} \circ(I+\mathbf{V}+\mathbf{W})$ and we have proved the claim. 
We now prove the existence of $\mathbf{W}^{*}(\mathbf{V})$. For this purpose, we introduce neighborhoods $\mathfrak{S}$ of $\Sigma$ and $\mathfrak{G}$ of $\Gamma$, such that $\mathfrak{S} \cap \mathfrak{G}=\emptyset$.

THEOREM 3.3 Assume that there exist two bounded open sets $\Omega, \omega$ of class $\mathrm{C}^{m+1, \alpha}, m \geqslant 2$, $0<\alpha<1$ such that the over-determined system (1.1)-(1.4) is satisfied in $\Omega \backslash \bar{\omega}$. Assume in addition that $\mathcal{H} \geqslant 0$ on $\Gamma=\partial \Omega$. Then there exists an open neighborhood $v$ of 0 in $\mathrm{e}_{b}^{m, \alpha}\left(\mathbb{R}^{2}, \mathbb{R}^{2}\right)$ and a function

$$
\text { V } \ni \mathbf{V} \mapsto \mathbf{W}^{*}(\mathbf{V}) \in \mathrm{C}_{b}^{m, \alpha}\left(\mathbb{R}^{2}, \mathbb{R}^{2}\right)
$$

of class $\mathrm{C}^{\infty}$ such that (1.1)-(1.4) has a solution in $\Omega_{\mathbf{W}^{*}(\mathbf{V})} \backslash \overline{\omega_{\mathbf{V}}}$ for all $\mathbf{V} \in V$ and $\mathbf{W}^{*}(0) \equiv 0$.

Proof. The main tool to prove this result is the implicit function theorem. First of all since $\Omega$ is of class $\mathrm{e}^{m+1, \alpha}$ we have $\mathcal{H}=\operatorname{div}_{\Gamma}(\mathbf{n}) \in \mathrm{e}^{m-1, \alpha}(\Gamma)$. Next, we introduce

$$
\begin{aligned}
& F: \mathrm{e}^{m, \alpha}(\Sigma) \times \mathrm{C}^{m, \alpha}(\Gamma) \rightarrow \mathrm{e}^{m, \alpha}(\bar{\Omega} \backslash \omega), \\
& \left(v_{n}, w_{n}\right) \mapsto\left(u_{1, \mathbf{V}, \mathbf{W}}-u_{2, \mathbf{v}, \mathbf{w}}\right) \circ(I+\mathbf{V}+\mathbf{W}),
\end{aligned}
$$

where

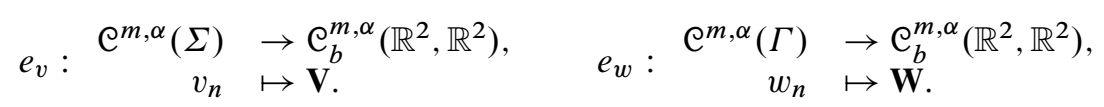

are linear extensions along the normal of $\left(v_{n}, w_{n}\right)$, i.e., $\left.\mathbf{V}\right|_{\Sigma}:=v_{n} \mathbf{n}_{\Sigma}$ and $\left.\mathbf{W}\right|_{\Gamma}:=w_{n} \mathbf{n}_{\Gamma}$, such that $\mathbf{V}$ has compact support in $\mathfrak{S}$ and $\mathbf{W}$ has compact support in $\mathfrak{G}$. Since $\Omega, \omega$ are of class $\mathbb{C}^{m+1, \alpha}$, we have $\mathbf{n}_{\Gamma} \in \mathrm{C}^{m, \alpha}(\Gamma)$ and $\mathbf{n}_{\Sigma} \in \mathrm{C}^{m, \alpha}(\Sigma)$ and we can find such extensions $e_{v}, e_{w}$. Since $(\mathbf{V}, \mathbf{W}) \in \mathfrak{C}$, it follows that $u_{1, \mathbf{V}, \mathbf{W}}$ and $u_{2, \mathbf{V}, \mathbf{W}} \in \mathrm{C}^{m, \alpha}\left(\overline{\Omega_{\mathbf{V}+\mathbf{W}}} \backslash \omega_{\mathbf{V}+\mathbf{W}}\right)=\mathrm{C}^{m, \alpha}\left(\overline{\Omega_{\mathbf{W}}} \backslash \omega_{\mathbf{V}}\right)$ due to the choice of extensions of $v_{n}$ and $w_{n}$.

Since we have assumed that there exist $\Omega$ and $\omega$ such that (1.1)-(1.4) has a solution, by uniqueness of the solution to (3.17)-(3.19) we get $u_{1,0,0}=u_{2,0,0}$ in $\bar{\Omega} \backslash \omega$ and therefore $F(0,0)=0$. From now on we write $u_{1}:=u_{1,0,0}$ and $u_{2}:=u_{2,0,0}$ for simplicity.

In order to apply the implicit function theorem and obtain $\mathbf{W}^{*}(\mathbf{V})$, we need to prove that $F$ is continuously differentiable, and that its derivative is an isomorphism. According to Lemma 3.2, we have

$$
(\mathbf{V}, \mathbf{W}) \in \mathfrak{C} \mapsto\left(u_{1, \mathbf{V}, \mathbf{W}}-u_{2, \mathbf{V}, \mathbf{w}}\right) \circ(I+\mathbf{V}+\mathbf{W}) \in \mathrm{e}^{m, \alpha}(\bar{\Omega} \backslash \omega)
$$

is of class $\mathrm{C}^{\infty}$ in a neighborhood of $(0,0)$. Since the extensions $e_{v}$ and $e_{w}$ are linear and continuous, we also get

$$
\left(v_{n}, w_{n}\right) \in \mathrm{C}^{m, \alpha}(\Sigma) \times \mathrm{C}^{m, \alpha}(\Gamma) \mapsto\left(u_{1, \mathbf{V}, \mathbf{W}}-u_{2, \mathbf{V}, \mathbf{w}}\right) \circ(I+\mathbf{V}+\mathbf{W}) \in \mathrm{C}^{m, \alpha}(\bar{\Omega} \backslash \omega)
$$

is of class $\mathrm{C}^{\infty}$. The derivative of $u_{i, 0, \mathbf{W}} \circ(I+\mathbf{W})$ in the direction $\widehat{\mathbf{W}}$ at $\mathbf{W}=0$ is the material derivative denoted $\dot{u}_{i}(\widehat{\mathbf{W}}), i=1,2$. Recall the definition of the shape derivative:

$$
u_{i}^{\prime}(\widehat{\mathbf{W}}):=\dot{u}_{i}(\widehat{\mathbf{W}})-\nabla u_{i} \cdot \widehat{\mathbf{W}}
$$

For a given test function $\hat{w}_{n}$ on $\Gamma$, we denote

$$
\widehat{\mathbf{W}}:=D_{w_{n}} e_{w}\left(0 ; \hat{w}_{n}\right)=e_{w}\left(\hat{w}_{n}\right),
$$


using linearity of $e_{w}$. We obtain due to $u_{1}=u_{2}$ :

$$
\begin{aligned}
D_{w_{n}} F\left(0,0 ; \hat{w}_{n}\right) & =\dot{u}_{1}(\widehat{\mathbf{W}})-\dot{u}_{2}(\widehat{\mathbf{W}})=u_{1}^{\prime}(\widehat{\mathbf{W}})-u_{2}^{\prime}(\widehat{\mathbf{W}})+\nabla u_{1} \cdot \widehat{\mathbf{W}}-\nabla u_{2} \cdot \widehat{\mathbf{W}} \\
& =u_{1}^{\prime}(\widehat{\mathbf{W}})-u_{2}^{\prime}(\widehat{\mathbf{W}}) .
\end{aligned}
$$

According to standard shape calculus; see [22, pp. 118-120] for instance, $u_{1}^{\prime}(\mathbf{W})$ and $u_{2}^{\prime}(\mathbf{W})$ satisfy the following equations:

$$
\begin{aligned}
& -\Delta u_{1}^{\prime}(\widehat{\mathbf{W}})=0 \quad \text { in } \quad \Omega \backslash \bar{\omega}, \\
& u_{1}^{\prime}(\widehat{\mathbf{W}})=0 \quad \text { on } \Sigma \text {, } \\
& u_{1}^{\prime}(\widehat{\mathbf{W}})=-\partial_{n} u_{1} \hat{w}_{n} \quad \text { on } \quad \Gamma \text {, }
\end{aligned}
$$

and

$$
\begin{aligned}
& -\Delta u_{2}^{\prime}(\widehat{\mathbf{W}})=0 \quad \text { in } \quad \Omega \backslash \bar{\omega}, \\
& u_{2}^{\prime}(\widehat{\mathbf{W}})=0 \quad \text { on } \Sigma, \\
& \partial_{n} u_{2}^{\prime}(\widehat{\mathbf{W}})=\operatorname{div}_{\Gamma}\left(\nabla_{\Gamma} u_{2} \hat{w}_{n}\right)+\mu \mathcal{H} \hat{w}_{n} \quad \text { on } \quad \Gamma,
\end{aligned}
$$

where we have taken into account the fact that $\widehat{\mathbf{W}}=e_{w}\left(\hat{w}_{n}\right)$ has compact support on $\mathfrak{G}$. We prove first the injectivity of $D_{w_{n}} F(0,0)$. Assume that $D_{w_{n}} F\left(0,0 ; \hat{w}_{n}\right)=0$. This implies $u_{1}^{\prime}(\widehat{\mathbf{W}})=$ $u_{2}^{\prime}(\widehat{\mathbf{W}})$. Taking into account that $u_{1}=u_{2}=0$ and $\partial_{n} u_{1}=\mu$ on $\Gamma$ as well, we get that $\hat{w}_{n}=$ $-\mu^{-1} u_{1}^{\prime}(\widehat{\mathbf{W}})$ on $\Gamma$ and $u_{2}^{\prime}(\widehat{\mathbf{W}})$ solves

$$
\begin{aligned}
-\Delta u_{2}^{\prime}(\widehat{\mathbf{W}})=0 & \text { in } \quad \Omega \backslash \bar{\omega}, \\
u_{2}^{\prime}(\widehat{\mathbf{W}})=0 & \text { on } \quad \Sigma, \\
\partial_{n} u_{2}^{\prime}(\widehat{\mathbf{W}})+\mathcal{H} u_{2}^{\prime}(\widehat{\mathbf{W}})=0 & \text { on } \quad \Gamma .
\end{aligned}
$$

Since $\mathcal{H} \in \mathrm{C}^{m-1, \alpha}(\Gamma)$ and $\mathcal{H} \geqslant 0$ on $\Gamma$, this function has a unique solution $u_{2}^{\prime}(\widehat{\mathbf{W}}) \equiv 0$ in view of Lemma 3.1. This implies $\hat{w}_{n}=0$ and the injectivity is proved.

Next, we prove surjectivity. Let $\psi \in \mathrm{e}^{m, \alpha}(\bar{\Omega} \backslash \omega)$. We are looking for a solution of the equation

$$
D_{w_{n}} F\left(0,0 ; \hat{w}_{n}\right)=u_{1}^{\prime}(\widehat{\mathbf{W}})-u_{2}^{\prime}(\widehat{\mathbf{W}})=\psi
$$

In view of the previous computation, $u_{2}^{\prime}(\widehat{\mathbf{W}})=u_{2}^{\prime}$ is independent of $\widehat{\mathbf{W}}$ and solves

$$
\begin{aligned}
-\Delta u_{2}^{\prime} & =0 & & \text { in } \quad \Omega \backslash \bar{\omega}, \\
u_{2}^{\prime} & =0 & & \text { on } \quad \Sigma, \\
\partial_{n} u_{2}^{\prime}+H u_{2}^{\prime} & =-H \psi & & \text { on } \quad \Gamma,
\end{aligned}
$$

and $w_{n}=-\mu^{-1}\left(u_{2}^{\prime}+\psi\right)$. Applying again Lemma 3.1, there exists a unique solution $u_{2}^{\prime} \in \mathrm{C}^{m, \alpha}(\bar{\Omega} \backslash$ $\omega)$. Consequently, we get

$$
\hat{w}_{n}=-\mu^{-1}\left(u_{2}^{\prime}+\psi\right) \in \mathrm{C}^{m, \alpha}(\Gamma),
$$

and this proves the surjectivity of $D_{w_{n}} F(0,0)$. 
We have shown that $D_{w_{n}} F(0,0)$ is an isomorphism from $\mathrm{e}^{m, \alpha}(\Gamma)$ to $\mathrm{C}^{m, \alpha}(\bar{\Omega} \backslash \omega)$. Therefore, we may apply the implicit function theorem to $F$, i.e., there exists a neighborhood $v_{\Gamma}$ of 0 in $\mathrm{e}^{m, \alpha}(\Sigma)$ and a unique $\mathrm{e}^{\infty}$ function

$$
\mathrm{e}^{m, \alpha}(\Sigma) \ni v_{n} \mapsto w_{n}^{*}\left(v_{n}\right) \in \mathrm{C}^{m, \alpha}(\Gamma)
$$

such that $F\left(v_{n}, w_{n}^{*}\left(v_{n}\right)\right) \equiv 0$ for all $v_{n} \in V_{\Gamma}$ and $w_{n}^{*}(0)=0$.

The statement of the theorem is obtained by considering the trace

$$
\begin{aligned}
r_{v}: \mathrm{C}_{b}^{m, \alpha}\left(\mathbb{R}^{2}, \mathbb{R}^{2}\right) & \rightarrow \mathrm{C}^{m, \alpha}(\Sigma), \\
\mathbf{V} & \left.\mapsto(\mathbf{V} \cdot \mathbf{n})\right|_{\Sigma}
\end{aligned}
$$

and the linear extension $e_{w}$. Note that the restriction $r_{v}$ is well-defined due to $\mathbf{n}_{\Sigma} \in \mathrm{e}^{m, \alpha}\left(\Sigma, \mathbb{R}^{2}\right)$. Taking a neighborhood $\bigcup$ of 0 in $\mathrm{C}_{b}^{m, \alpha}\left(\mathbb{R}^{2}, \mathbb{R}^{2}\right)$ such that $r_{v}(\mathcal{V}) \subset V_{\Gamma}$ and applying the previous result with $v_{n}:=r_{v}(\mathbf{V})$, we get a unique $w_{n}^{*}$ in $\mathrm{C}^{m, \alpha}(\Gamma)$. Setting $\mathbf{W}^{*}:=e_{w}\left(w_{n}^{*}\right)$ we obtain the main statement. Since the extension $e_{w}$ is obviously not unique, $\mathbf{W}^{*}$ is not unique as well, even if $w_{n}^{*}$ is. We can also note that $\mathbf{W}^{*}$ depends actually only on $v_{n}=\left.(\mathbf{V} \cdot \mathbf{n})\right|_{\Gamma}$ and not on its extension V.

Finally, the trace $r_{v}$ and extension $e_{w}$ are linear and continuous. Therefore the function

$$
\text { V } \mathbf{V} \mapsto \mathbf{W}^{*}(\mathbf{V}) \in \mathrm{C}_{b}^{m, \alpha}\left(\mathbb{R}^{2}, \mathbb{R}^{2}\right)
$$

is of class $\mathrm{e}^{\infty}$ by composition.

COROLLARY 3.4 Under the same assumptions as in Theorem 3.3, the derivative of $\mathbf{W}^{*}(\mathbf{V})$ in direction $\widehat{\mathbf{V}}$ at $\mathbf{V}=0$ in $\mathbb{C}_{b}^{m, \alpha}\left(\mathbb{R}^{2}, \mathbb{R}^{2}\right)$ is such that

$$
D_{\mathbf{V}} \mathbf{W}^{*}(0 ; \widehat{\mathbf{V}})=-\mu^{-1} \bar{u}(\widehat{\mathbf{V}}) \mathbf{n}_{\Gamma} \text { on } \Gamma,
$$

where $\bar{u}(\widehat{\mathbf{V}})$ is the solution of

$$
\begin{aligned}
& -\Delta \bar{u}(\widehat{\mathbf{V}})=0 \quad \text { in } \quad \Omega \backslash \bar{\omega}, \\
& \bar{u}(\widehat{\mathbf{V}})=-\partial_{n} u \widehat{\mathbf{V}} \cdot \mathbf{n}_{\Sigma} \quad \text { on } \quad \Sigma, \\
& \partial_{n} \bar{u}(\widehat{\mathbf{V}})+\mathcal{H} \bar{u}(\widehat{\mathbf{V}})=0 \quad \text { on } \quad \Gamma,
\end{aligned}
$$

where $\Gamma=\Gamma^{*}(\omega)$ and $\Omega=\Omega^{*}(\omega)$.

Proof. By Theorem 3.3, there exists a neighborhood $\bigcup_{\Gamma}$ of 0 in $\mathrm{C}^{m, \alpha}(\Sigma)$ and a unique $\mathrm{C}^{\infty}$ function

$$
\mathrm{C}^{m, \alpha}(\Sigma) \ni v_{n} \mapsto w_{n}^{*}\left(v_{n}\right) \in \mathrm{C}^{m, \alpha}(\Gamma)
$$

such that $F\left(v_{n}, w_{n}^{*}\left(v_{n}\right)\right) \equiv 0$ for all $v_{n} \in V_{\Gamma}$ and $w_{n}^{*}(0)=0$. Differentiating $F\left(v_{n}, w_{n}^{*}\left(v_{n}\right)\right)=0$ with respect to $v_{n}$ in direction $\hat{v}_{n}$ one obtains

$$
D_{w_{n}} F\left(0,0 ; \hat{w}_{n}\right)+D_{v_{n}} F\left(0,0 ; \hat{v}_{n}\right)=0,
$$

where $\hat{w}_{n}:=D_{v_{n}} w_{n}^{*}\left(0 ; \hat{v}_{n}\right)$. This yields

$$
\hat{w}_{n}=-\left(D_{w_{n}} F(0,0)\right)^{-1} D_{v_{n}} F\left(0,0 ; \hat{v}_{n}\right)
$$


since $D_{w_{n}} F(0,0)$ is an isomorphism. We prove that $\hat{w}_{n}=-\left.\mu^{-1} \bar{u}(\widehat{\mathbf{V}})\right|_{\Gamma}$ is solution of (3.33). Indeed, choosing $\hat{w}_{n}=-\left.\mu^{-1} \bar{u}(\widehat{\mathbf{V}})\right|_{\Gamma}$, we have

$$
\begin{aligned}
D_{w_{n}} F\left(0,0 ; \hat{w}_{n}\right) & =u_{1}^{\prime}(\widehat{\mathbf{W}})-u_{2}^{\prime}(\widehat{\mathbf{W}}), \\
D_{v_{n}} F\left(0,0 ; \hat{v}_{n}\right) & =u_{1}^{\prime}(\widehat{\mathbf{V}})-u_{2}^{\prime}(\widehat{\mathbf{V}}),
\end{aligned}
$$

where $u_{1}^{\prime}(\widehat{\mathbf{W}}), u_{2}^{\prime}(\widehat{\mathbf{W}}), u_{1}^{\prime}(\widehat{\mathbf{V}}), u_{2}^{\prime}(\widehat{\mathbf{V}})$ are harmonic functions on $\Omega \backslash \bar{\omega}$, with the boundary conditions $u_{1}^{\prime}(\widehat{\mathbf{W}})=u_{2}^{\prime}(\widehat{\mathbf{W}})=0$ on $\Sigma$ and $u_{1}^{\prime}(\widehat{\mathbf{V}})=\partial_{n} u_{2}^{\prime}(\widehat{\mathbf{V}})=0$ on $\Gamma$. Moreover, we have $u_{1}^{\prime}(\widehat{\mathbf{W}})=-\mu \hat{w}_{n}$ and $\partial_{n} u_{2}^{\prime}(\widehat{\mathbf{W}})=\mu \mathcal{H} \hat{w}_{n}$ on $\Gamma$ and $u_{1}^{\prime}(\widehat{\mathbf{V}})=u_{2}^{\prime}(\widehat{\mathbf{V}})=-\partial_{n} u \hat{v}_{n}$ on $\Sigma$. Now define

$$
\begin{aligned}
\tilde{u}(\widehat{\mathbf{W}}) & :=u_{1}^{\prime}(\widehat{\mathbf{W}})-u_{2}^{\prime}(\widehat{\mathbf{W}}), \\
\tilde{u}(\widehat{\mathbf{V}}) & :=u_{1}^{\prime}(\widehat{\mathbf{V}})-u_{2}^{\prime}(\widehat{\mathbf{V}})
\end{aligned}
$$

We then have

$$
\begin{aligned}
-\Delta \tilde{u}(\widehat{\mathbf{W}})=0 & \text { in } \quad \Omega \backslash \bar{\omega}, \\
\tilde{u}(\widehat{\mathbf{W}})=0 & \text { on } \quad \Sigma, \quad \tilde{u}(\widehat{\mathbf{W}})=\bar{u}(\widehat{\mathbf{V}})-u_{2}^{\prime}(\widehat{\mathbf{W}}) \quad \text { on } \quad \Gamma,
\end{aligned}
$$

and

$$
\begin{aligned}
-\Delta \tilde{u}(\widehat{\mathbf{V}})=0 & \text { in } \quad \Omega \backslash \bar{\omega}, \\
\tilde{u}(\widehat{\mathbf{V}})=0 & \text { on } \quad \Sigma, \quad \tilde{u}(\widehat{\mathbf{V}})=-u_{2}^{\prime}(\widehat{\mathbf{V}}) \quad \text { on } \quad \Gamma .
\end{aligned}
$$

The difference $\bar{u}(\widehat{\mathbf{V}})-u_{2}^{\prime}(\widehat{\mathbf{W}})$ satisfies the equation

$$
\begin{aligned}
& -\Delta\left(\bar{u}(\widehat{\mathbf{V}})-u_{2}^{\prime}(\widehat{\mathbf{W}})\right)=0 \quad \text { in } \quad \Omega \backslash \bar{\omega}, \\
& \bar{u}(\widehat{\mathbf{V}})-u_{2}^{\prime}(\widehat{\mathbf{W}})=-\partial_{n} u \hat{v}_{n} \quad \text { on } \quad \Sigma, \\
& \partial_{n}\left(\bar{u}(\widehat{\mathbf{V}})-u_{2}^{\prime}(\widehat{\mathbf{W}})\right)=-\mathcal{H} \bar{u}(\widehat{\mathbf{V}})-\mu \mathcal{H} \hat{w}_{n}=0 \quad \text { on } \Gamma \text {, }
\end{aligned}
$$

where we have used $\hat{w}_{n}=-\left.\mu^{-1} \bar{u}(\widehat{\mathbf{V}})\right|_{\Gamma}$. Thus we observe that $\bar{u}(\widehat{\mathbf{V}})-u_{2}^{\prime}(\widehat{\mathbf{W}})=u_{2}^{\prime}(\widehat{\mathbf{V}})$. This yields in view of (3.34)

$$
\begin{aligned}
-\Delta \tilde{u}(\widehat{\mathbf{W}})=0 & \text { in } \quad \Omega \backslash \bar{\omega}, \\
\tilde{u}(\widehat{\mathbf{W}})=0 & \text { on } \quad \Sigma, \quad \tilde{u}(\widehat{\mathbf{W}})=u_{2}^{\prime}(\widehat{\mathbf{V}}) \quad \text { on } \quad \Gamma .
\end{aligned}
$$

It follows from (3.35) and (3.36) that $\tilde{u}(\widehat{\mathbf{W}})=-\tilde{u}(\widehat{\mathbf{V}})$ which means $D_{w_{n}} F\left(0,0 ; \hat{w}_{n}\right)=$ $-D_{v_{n}} F\left(0,0 ; \hat{v}_{n}\right)$, and that $\hat{w}_{n}=-\left.\mu^{-1} \bar{u}(\widehat{\mathbf{V}})\right|_{\Gamma}$ is indeed the solution of (3.33). Since the extension $e_{w}$ and the restriction $r_{v}$ from the proof of Theorem 3.3 are linear, we obtain

$$
D_{\mathbf{V}} \mathbf{W}^{*}(0 ; \widehat{\mathbf{V}})=e_{w}\left(D_{v_{n}} w_{n}^{*}\left(0 ; r_{v}(\widehat{\mathbf{V}})\right)\right)
$$

which yields

$$
D_{\mathbf{V}} \mathbf{W}^{*}(0 ; \widehat{\mathbf{V}})=-\mu^{-1} \bar{u}(\widehat{\mathbf{V}}) \mathbf{n}_{\Gamma} \quad \text { on } \Gamma \text {. }
$$


In view of Corollary 3.4 we define $\widehat{\mathbf{W}}^{*}:=D_{\mathbf{V}} \mathbf{W}^{*}(0 ; \widehat{\mathbf{V}})$. From (3.9)-(3.11) we may deduce a local monotonicity result.

THEOREM 3.5 Let $\Omega$ and $\omega$ satisfy assumptions as in Theorem 3.3 and let $\widehat{\mathbf{V}} \in \mathcal{C}_{b}^{m, \alpha}\left(\mathbb{R}^{2}, \mathbb{R}^{2}\right)$, $m \geqslant 2$. Assume $\mu<0, \widehat{\mathbf{V}}(x) \cdot \mathbf{n}(x) \leqslant 0$ for all $x \in \Sigma$ and there exists $x \in \Sigma$ such that $\widehat{\mathbf{V}}(x) \cdot \mathbf{n}(x)<0$, then $\widehat{\mathbf{W}}^{*}(x) \cdot \mathbf{n}(x)>0$ for all $x \in \Gamma$.

Proof. Since $\bar{u}(\widehat{\mathbf{V}})$ is harmonic, the maximum principle states that the minimum of $\bar{u}(\widehat{\mathbf{V}})$ is attained on $\Gamma \cup \Sigma$. The function $u$ is also harmonic. Therefore, its maximum is attained on $\Sigma$, where $u=1$ and $\partial_{n} u(x)>0$ for all $x \in \Sigma$. Therefore, in view of the assumption $\widehat{\mathbf{V}} \cdot \mathbf{n} \leqslant 0$ on $\Sigma$ we have $\bar{u}(\widehat{\mathbf{V}}) \geqslant 0$ on $\Sigma$ due to (3.31). Consequently, if $\bar{u}(\widehat{\mathbf{V}})$ takes negative values on $\Gamma$ then the minimum of $\bar{u}(\widehat{\mathbf{V}})$ is attained on $\Gamma$.

Due to (3.29) and since $\mu<0$ by assumption, the claim $\widehat{\mathbf{W}}^{*}(x) \cdot \mathbf{n}(x)>0$ for all $x \in \Gamma$ amounts to proving that $\bar{u}(\widehat{\mathbf{V}})>0$ on $\Gamma$. By contradiction, we assume that there exists a point $z \in \Gamma$ such that $\bar{u}(\widehat{\mathbf{V}})(z) \leqslant 0$. In this case, we have shown that the minimum of $\bar{u}(\widehat{\mathbf{V}})$ is attained on $\Gamma$ so we may assume that $z \in \Gamma$ is precisely the minimizer, and we assume in addition that $\bar{u}(\widehat{\mathbf{V}})(z)<\bar{u}(\widehat{\mathbf{V}})(x)$ for all $x \in \Omega$. Since $\Omega$ is convex, we have $\mathcal{H}(z) \geqslant 0$ for all $z \in \Gamma$. Therefore, due to (3.32), we have

$$
\partial_{n} \bar{u}(\widehat{\mathbf{V}})(z)=-\mathcal{H}(z) \bar{u}(\widehat{\mathbf{V}})(z) \geqslant 0 .
$$

Since $\Omega$ is at least of class $\mathrm{C}^{3, \alpha}$, it satisfies the interior ball condition and we may apply Hopf's lemma (see [20] for details), implying that $\partial_{n} \bar{u}(\widehat{\mathbf{V}})(z)<0$ in contradiction with (3.37). Thus the initial assumption cannot be satisfied and either $\bar{u}(\widehat{\mathbf{V}})(z)>0$ for all $z \in \Gamma$ or $\bar{u}(\widehat{\mathbf{V}})(z) \leqslant 0$ and there exists $x \in \Omega$ such that $\bar{u}(\widehat{\mathbf{V}})(z)=\bar{u}(\widehat{\mathbf{V}})(x)$. In the second case, $\bar{u}(\widehat{\mathbf{V}})$ must be constant in $\Omega$ due to the strong maximum principle and since $\bar{u}(\widehat{\mathbf{V}})(z) \geqslant 0$ on $\Sigma$ we get $\bar{u}(\widehat{\mathbf{V}}) \equiv 0$ in $\bar{\Omega}$ which leads to $\widehat{\mathbf{V}} \cdot \mathbf{n} \equiv 0$ on $\Sigma$, a property excluded by assumption. Therefore, $\bar{u}(\widehat{\mathbf{V}})>0$ on $\Gamma$ and $\widehat{\mathbf{W}}^{*}(x) \cdot \mathbf{n}(x)>0$ for all $x \in \Gamma$ in view of (3.29).

REMARK 3.6 The convexity of $\Omega$ in Theorem 3.5 holds whenever $\omega$ is convex (See for instance [13, Theorem 6.2.2])

REMARK 3.7 Under the assumptions of Theorem 3.3, Theorem 3.5 leads to the monotonicity for the set inclusion of $\Omega^{*}(\omega)$ with respect to a convex $\omega$ for small perturbations of $\omega$, i.e., if $\omega_{1} \subset \omega_{2}$ are two convex sets and $\omega_{2}$ is close to $\omega_{1}$ in the sense that there exists a $\mathbf{V} \in V$ with $\mathbf{V} \cdot \mathbf{n} \leqslant 0$ on $\Sigma_{1}=\partial \omega_{1}$ such that $\omega_{2}=(I+\mathbf{V})\left(\omega_{1}\right)$ (note that $\mathbf{n}$ denotes here the inner normal vector to $\left.\omega_{1}\right)$, then $\Omega^{*}\left(\omega_{1}\right) \subset \Omega^{*}\left(\omega_{2}\right)$. Indeed, we then have $\Omega^{*}\left(\omega_{2}\right)=\left(I+\mathbf{W}^{*}(\mathbf{V})\right)\left(\Omega^{*}\left(\omega_{1}\right)\right)$ and using Theorem 3.5 we get $D_{\mathbf{V}} \mathbf{W}^{*}(0 ; \mathbf{V}) \cdot \mathbf{n}>0$. Since for a small perturbation $\mathbf{V}$ we have

$$
\mathbf{W}^{*}(\mathbf{V})=\mathbf{W}^{*}(0)+D_{\mathbf{V}} \mathbf{W}^{*}(0 ; \mathbf{V})+o\left(\|\mathbf{V}\|_{m, \alpha}^{2}\right)
$$

and $\mathbf{W}^{*}(0)=0$, we can choose $\omega_{2}$ and $\mathbf{V}$ small enough such that $\mathbf{W}^{*}(\mathbf{V}) \cdot \mathbf{n} \geqslant 0$ and $\Omega^{*}\left(\omega_{1}\right) \subset$ $\Omega^{*}\left(\omega_{2}\right)$ follows.

In what follows, we will need the following standard lemma.

Lemma 3.8 Let $\omega$ be of class $\mathbb{C}^{m, \alpha}, \mathbf{V} \in \mathbb{C}_{b}^{m, \alpha}\left(\mathbb{R}^{2}, \mathbb{R}^{2}\right), m \geqslant 2$ and $\omega_{\mathbf{V}}=(I+\mathbf{V})(\omega)$. Let $f$ be such that $\mathbf{V} \rightarrow f(\mathbf{V}) \circ(I+\mathbf{V}) \in L^{1}(\omega)$ is differentiable at $\mathbf{V}=0$ from $\mathrm{C}_{b}^{m, \alpha}\left(\mathbb{R}^{2}, \mathbb{R}^{2}\right)$ to $L^{1}(\omega)$ with derivative $f^{\prime}(0)$ and $f(0) \in W^{1,1}\left(\mathbb{R}^{2}\right)$. Consider the functional

$$
J(\mathbf{V})=\int_{\omega_{\mathbf{V}}} f(\mathbf{V}) d x .
$$


Then

$$
D_{\mathbf{V}} J(0 ; \widehat{\mathbf{V}})=\int_{\omega} f^{\prime}(0 ; \widehat{\mathbf{V}})+\operatorname{div}(f(0) \widehat{\mathbf{V}}) d x=\int_{\omega} f^{\prime}(0 ; \widehat{\mathbf{V}}) d x+\int_{\partial \omega} f(0) \widehat{\mathbf{V}} \cdot \mathbf{n} d s,
$$

where $f^{\prime}(0 ; \widehat{\mathbf{V}})$ is the shape derivative of $f(\mathbf{V})$.

Proof. The result is a straightforward consequence of, for instance, [18, Theorem 4.1].

\subsection{Shape derivative of the cost functional $K_{1}$}

We express the shape derivative of $K_{1}$ as defined in (3.1) in the Hadamard-Zolésio structure form (3.3), under appropriate smoothness conditions on the boundary of $\Omega$.

THEOREM 3.9 Let $\omega \subset \mathbb{R}^{2}$ be a bounded domain, with a boundary of class $\mathrm{C}^{2, \alpha}$, and let $\mathbf{V} \in$ $\mathrm{C}_{b}^{2, \alpha}\left(\mathbb{R}^{2}, \mathbb{R}^{2}\right)$ be given. Assume $\mathcal{H} \geqslant 0$ on $\Gamma^{*}(\omega)$. Then the shape gradient $\nabla K_{1}(\omega)$ of the cost $K_{1}$ can be expressed as

$$
\nabla K_{1}(\omega)=\nabla p \cdot \nabla u \in \mathrm{C}^{1, \alpha}(\Sigma),
$$

where all expressions are evaluated on $\Sigma$, and the adjoint state $p$ satisfies

$$
\begin{aligned}
& -\Delta p=0 \quad \text { in } \Omega^{*}(\omega) \backslash \bar{\omega}, \\
& p=0 \quad \text { on } \Sigma \text {, } \\
& \partial_{n} p+H p=-\mu^{-1} \mathbb{1}_{E^{c}}+\mu^{-1} \mathbb{1}_{E} \text { on } \quad \Gamma^{*}(\omega) \text {. }
\end{aligned}
$$

Proof. Using Lemma 3.8 we obtain for $\mathbf{W} \in \mathrm{C}_{b}^{2, \alpha}\left(\mathbb{R}^{2}, \mathbb{R}^{2}\right)$ the shape derivative of $J_{1}(\Omega)$ at $\Omega$ :

$$
d J_{1}(\Omega ; \mathbf{W})=\int_{\Gamma \cap E^{c}} \mathbf{W} \cdot \mathbf{n} d s+\int_{\Gamma \cap E}-\mathbf{W} \cdot \mathbf{n} d s
$$

Note that in (3.42), there is no contribution from the shape derivative along $\partial E$ since $E$ is fixed. The minus sign in the second integral comes from the orientation of the normal vector in $E \cap \Omega^{c}$. Since $K_{1}\left(\omega_{\mathbf{V}}\right)=J_{1}\left(\left(I+\mathbf{W}^{*}(\mathbf{V})\right)\left(\Omega^{*}(\omega)\right)\right)$ we may apply the chain rule thanks to Theorem 3.3, Corollary 3.4 and (3.42). Using (3.29) we obtain

$$
\begin{aligned}
d K_{1}(\omega ; \widehat{\mathbf{V}}) & =d J_{1}\left(\Omega^{*}(\omega) ; D_{\mathbf{V}} \mathbf{W}^{*}(0 ; \widehat{\mathbf{V}})\right) \\
& =\int_{\Gamma^{*} \cap E^{c}}-\mu^{-1} u^{\prime} d s+\int_{\Gamma^{*} \cap E} \mu^{-1} u^{\prime} d s=\int_{\Gamma^{*}}\left(-\mu^{-1} \mathbb{1}_{E^{c}}+\mu^{-1} \mathbb{1}_{E}\right) u^{\prime} d s
\end{aligned}
$$

To simplify (3.43), we introduce the adjoint state $p$ solution of (3.39)-(3.41), which is well-defined due to $\mathcal{H} \geqslant 0$ on $\Gamma^{*}(\omega)$. Using Green's formula in $\Omega \backslash \bar{\omega}$ and utilizing (3.39)-(3.41), we obtain

$$
\begin{aligned}
\int_{\Gamma^{*}}\left(-\mu^{-1} \mathbb{1}_{E^{c}}+\mu^{-1} \mathbb{1}_{E}\right) u^{\prime} d s & =\int_{\Gamma^{*}}\left(\partial_{n} p+\mathcal{H} p\right) u^{\prime} d s \\
= & \int_{\Omega^{*} \backslash \bar{\omega}}\left(\Delta p u^{\prime}-p \Delta u^{\prime}\right) d x+\int_{\Gamma^{*}} p\left(\partial_{n} u^{\prime}+\mathcal{H} u^{\prime}\right) d s \\
& +\int_{\Sigma}\left(-u^{\prime} \partial_{n} p+p \partial_{n} u^{\prime}\right) d s .
\end{aligned}
$$


Using (3.13)-(3.15) and (3.39)-(3.41) yields

$$
\int_{\Gamma^{*}}\left(-\mu^{-1} \mathbb{1}_{E^{c}}+\mu^{-1} \mathbb{1}_{E}\right) u^{\prime} d s=\int_{\Sigma}\left(\partial_{n} p \partial_{n} u\right) \widehat{\mathbf{V}} \cdot \mathbf{n} d s=\int_{\Sigma} \nabla p \cdot \nabla u \widehat{\mathbf{V}} \cdot \mathbf{n} d s .
$$

Since $\omega$ is of class $\mathrm{C}^{2, \alpha}$ and $\Omega^{*} \backslash \omega$ is of class $\mathrm{C}^{\infty}$ due to assumption (2.4), we have $\left.p\right|_{\Sigma},\left.u\right|_{\Sigma} \in$ $\mathrm{e}^{2, \alpha}(\Sigma)$ due to standard regularity results and $\mathbf{n}_{\Sigma} \in \mathrm{e}^{1, \alpha}\left(\Sigma, \mathbb{R}^{2}\right)$. Therefore, $\nabla K_{1}(\omega)=\nabla p \cdot \nabla u \in$ $\mathrm{e}^{1, \alpha}(\Sigma)$ and

$$
d K_{1}(\omega ; \widehat{\mathbf{V}})=\int_{\Sigma} \nabla p \cdot \nabla u \widehat{\mathbf{V}} \cdot \mathbf{n} d s
$$

is well-defined.

\subsection{Shape derivative of the cost functional $K_{2}$}

In a similar way we express the shape derivative of $K_{2}$ in the Hadamard-Zolésio structure form (3.3), under appropriate smoothness conditions on the boundary of $\Omega$.

THEOREM 3.10 Let $\omega \subset \mathbb{R}^{2}$ be a bounded domain, with a boundary of class $\mathrm{C}^{2, \alpha}$, and let $\mathbf{V} \in$ $\mathrm{C}_{b}^{2, \alpha}\left(\mathbb{R}^{2}, \mathbb{R}^{2}\right)$ be given. Assume $\mathcal{H} \geqslant 0$ on $\Gamma^{*}(\omega)$. Then the shape derivative of $K_{2}$ at $\omega$ in direction $\widehat{\mathbf{V}}$ is

$$
d K_{2}(\omega, \widehat{\mathbf{V}})=\int_{\Sigma}\left[\nabla u \cdot \nabla p+\nabla p_{l} \cdot \nabla u_{l}\right] \widehat{\mathbf{V}} \cdot \mathbf{n} d s,
$$

and the adjoint states $p_{l}$ and $p$ satisfy

$$
\begin{aligned}
-\Delta p_{l} & =-\left(u-u_{l}\right) & & \text { in } E \backslash \bar{\omega}, \\
p_{l} & =0 & & \text { on } \Sigma, \\
p_{l} & =0 & & \text { on } \partial E,
\end{aligned}
$$

and

$$
\begin{aligned}
-\Delta p & =u \mathbb{1}_{\Omega^{*}(\omega) \cap E^{c}}+\left(u-u_{l}\right) \mathbb{1}_{E \cap \omega^{c}} & & \text { in } \Omega^{*}(\omega) \backslash \bar{\omega}, \\
p & =0 & & \text { on } \Sigma, \\
\partial_{n} p+\mathcal{H} p & =0 & & \text { on } \Gamma^{*}(\omega),
\end{aligned}
$$

respectively.

Proof. In view of Lemma (3.2) the conditions of Lemma 3.8 are fulfilled and we may apply the chain rule to $K_{2}(\omega)$ thanks to Theorem 3.3 and Corollary 3.4. Note that $J_{2}$ depends on two variables $\Omega$ and $\omega$, therefore we obtain the shape derivative of $K_{2}(\omega)$ with respect to $\omega$ in direction $\widehat{\mathbf{V}}$ as

$$
\begin{aligned}
d K_{2}(\omega ; \widehat{\mathbf{V}})= & d J_{2}\left(\Omega^{*}(\omega), \omega ; D_{\mathbf{V}} \mathbf{W}^{*}(0 ; \widehat{\mathbf{V}}), \widehat{\mathbf{V}}\right) \\
= & \int_{\Omega^{*} \cap E^{c}} u u^{\prime}(\widehat{\mathbf{V}}) d x+\int_{E \cap \omega^{c}}\left(u-u_{l}\right)\left(u^{\prime}(\widehat{\mathbf{V}})-u_{l}^{\prime}(\widehat{\mathbf{V}})\right) d x \\
& +\frac{1}{2} \int_{\Gamma^{*} \cap E^{c}} u^{2} D_{\mathbf{V}} \mathbf{W}^{*}(0 ; \widehat{\mathbf{V}}) \cdot \mathbf{n} d s+\frac{1}{2} \int_{\Sigma}\left(u-u_{l}\right)^{2} \widehat{\mathbf{V}} \cdot \mathbf{n} d s
\end{aligned}
$$


where $u^{\prime}=u^{\prime}(\widehat{\mathbf{V}})$ is the solution of (3.13)-(3.15) and $u_{l}^{\prime}=u_{l}^{\prime}(\widehat{\mathbf{V}})$ satisfies [22, p. 118]

$$
\begin{aligned}
& -\Delta u_{l}^{\prime}=0 \quad \text { in } E \backslash \bar{\omega} \text {, } \\
& u_{l}^{\prime}=-\partial_{n} u_{l} \widehat{\mathbf{V}} \cdot \mathbf{n} \quad \text { on } \Sigma \text {, } \\
& u_{l}^{\prime}=0 \quad \text { on } \partial E \text {. }
\end{aligned}
$$

In view of the boundary conditions for $u$ and $u_{l}$ we get

$$
\begin{aligned}
d K_{2}(\omega ; \widehat{\mathbf{V}}) & =\int_{\Omega^{*} \cap E^{c}} u u^{\prime} d x+\int_{E \cap \omega^{c}}\left(u-u_{l}\right)\left(u^{\prime}-u_{l}^{\prime}\right) d x \\
& =\int_{\Omega^{*} \cap \omega^{c}}\left(u \mathbb{1}_{\Omega^{*} \cap E^{c}}+\left(u-u_{l}\right) \mathbb{1}_{E \cap \omega^{c}}\right) u^{\prime} d x+\int_{E \cap \omega^{c}}-\left(u-u_{l}\right) u_{l}^{\prime} d x,
\end{aligned}
$$

using $u^{\prime} \equiv 0$ on $\left(\Omega^{*}(\omega)\right)^{c}$. Next, we simplify the second integral in (3.57) by introducing the adjoint state $p_{l}$, the solution to (3.47)-(3.49), and we obtain

$$
\begin{aligned}
\int_{E \cap \omega^{c}}-\left(u-u_{l}\right) u_{l}^{\prime} d x & =\int_{E \cap \omega^{c}}-\Delta p_{l} u_{l}^{\prime} d x \\
& =\int_{E \cap \omega^{c}}-p_{l} \Delta u_{l}^{\prime} d x+\int_{\Sigma}\left(-\partial_{n} p_{l} u_{l}^{\prime}+p_{l} \partial_{n} u_{l}^{\prime}\right) d s \\
& =\int_{\Sigma} \partial_{n} p_{l} \partial_{n} u_{l} \widehat{\mathbf{v}} \cdot \mathbf{n} d s=\int_{\Sigma} \nabla p_{l} \cdot \nabla u_{l} \widehat{\mathbf{v}} \cdot \mathbf{n} d s .
\end{aligned}
$$

Similarly, to simplify the first integral in (3.57), we introduce the adjoint state $p$ solution of (3.50)(3.52), which leads to

$$
\begin{aligned}
\int_{\Omega^{*} \cap \omega^{c}}\left(u \mathbb{1}_{\Omega^{*} \cap E^{c}}+\left(u-u_{l}\right) \mathbb{1}_{\Omega^{*} \cap \omega^{c}}\right) u^{\prime} d x & =\int_{\Omega^{*} \cap \omega^{c}}-\Delta p u^{\prime} d x \\
& =\int_{\Omega^{*} \cap \omega^{c}}-p \Delta u^{\prime} d x \\
& +\int_{\Gamma^{*} \cup \Sigma}\left(-\partial_{n} p u^{\prime}+p \partial_{n} u^{\prime}\right) d s \\
& =\int_{\Sigma} \partial_{n} p \partial_{n} u \widehat{\mathbf{V}} \cdot \mathbf{n} d s+\int_{\Gamma^{*}} u^{\prime}\left(-\partial_{n} p-\mathcal{H} p\right) d s \\
& =\int_{\Sigma} \partial_{n} p \partial_{n} u \widehat{\mathbf{V}} \cdot \mathbf{n} d s=\int_{\Sigma} \nabla u \cdot \nabla p \widehat{\mathbf{V}} \cdot \mathbf{n} d s,
\end{aligned}
$$

where we used (3.15). Since the mapping $d K_{2}(\omega ; \widehat{\mathbf{V}})$ is linear and continuous, we have obtained (3.46).

\section{Numerical algorithm and examples}

We solve the optimization problems using an iterative process, i.e., we find a solution to the lowerlevel problem $\left(\mathcal{F}_{\omega}\right)$ first and then proceed to the upper-level problem consisting of the minimization of $K_{1}$ and $K_{2}$. For the upper-level problem, we use the boundary variation technique [2]. One may 
use the negative shape gradients $\mathbf{V}_{i}=-\nabla K_{i}(\omega) \mathbf{n}$ on $\Sigma, i=1,2$ as a descent direction, which need to be extended to the entire domain for the numerical method. In Algorithm 2 we introduce an extension of $\mathbf{V}_{i}$ over the entire domain $\Omega^{*} \backslash \bar{\omega}$ such that

$$
d K_{i}\left(\omega ; \mathbf{V}_{i}\right)=\int_{\Sigma} \nabla K_{i}(\omega) \mathbf{V}_{i} \cdot \mathbf{n} d s=-\int_{\Omega^{*} \backslash \omega}\left|D \mathbf{V}_{i}\right|^{2}+\left|\mathbf{V}_{i}\right|^{2} d \mathbf{x}<0
$$

which yields a descent direction for the cost functionals $K_{i}, i=1,2$.

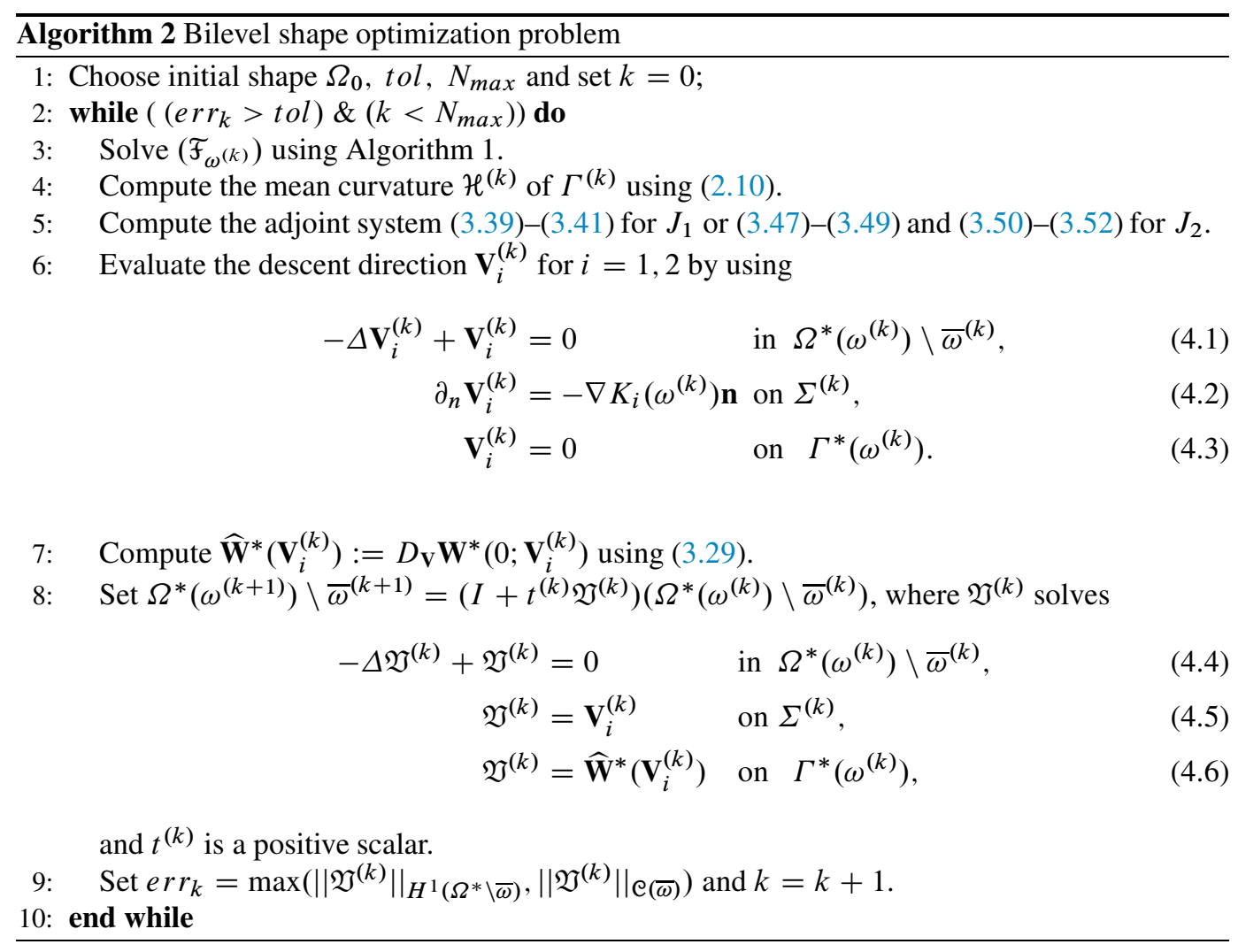

In step (8) of Algorithm 2, both sets $\omega$ and $\Omega^{*}(\omega)$ are updated. However, the actual update for the free set $\Omega^{*}(\omega)$ occurs in step (3). The purpose of the update of $\Omega^{*}(\omega)$ in step (8) is not to solve the free boundary problem but to provide a good initialization for solving $\left(\mathcal{F}_{\omega}\right)$ later in step (3). Alternatively, it would be sufficient to update $\omega$ only, on the basis of the vector field $\mathbf{V}_{i}$ obtained in step (6), hence avoiding the computation of $\mathfrak{V}$ and the update of $\Omega^{*}(\omega)$, but numerical experience shows that step (8) is advantageous as it allows to decrease the amount of iterations in step (3).

The current form of Algorithm 2 does not satisfy the inequality constraints in $\boldsymbol{U}_{a d}$. To achieve these constraints, a penalty approach is used. Furthermore, the extension $\mathbf{V}_{i}$ of $-\nabla K_{i}(\omega) \mathbf{n}$ on the basis of (4.1)-(4.3) is also regularizing. Namely, if $\omega$ is of class $\mathrm{C}^{2, \alpha}$ we have shown for instance in (3.38) that $\nabla K_{1}(\omega) \in \mathrm{C}^{1, \alpha}(\Sigma)$ and the extension $\mathbf{V}_{i}$ is in $\mathrm{C}^{2, \alpha}\left(\overline{\Omega^{*}(\omega)} \backslash \omega\right)$ in view of (4.1)(4.3). If the Neumann boundary condition in (4.2) is replaced by a Dirichlet condition, then the 
regularization is insufficient and undesired oscillations of the shapes may occur [15].

Note that the shape gradient in (3.46) for the reduced cost $K_{2}$ involves the functions $u, p$ and $u_{l}, p_{l}$ defined on domains $\Omega^{*}(\omega) \backslash \bar{\omega}$ and $E \backslash \bar{\omega}$, respectively. From the numerical implementation perspective, these domains and the associated grids must be updated separately and consequently, some modification of Algorithm 2 is necessary. Specifically, in addition to steps (4.1)-(4.6) in Algorithm 2, the domain $E \backslash \bar{\omega}$ is updated via

$$
E \backslash \bar{\omega}^{(k+1)}=\left(I+t^{(k)} \mathfrak{W}^{(k)}\right)\left(E \backslash \bar{\omega}^{(k)}\right),
$$

where $\mathfrak{W}^{(k)}$ solves

$$
\begin{aligned}
-\Delta \mathfrak{W}^{(k)}+\mathfrak{W}^{(k)} & =0 & & \text { in } E \backslash \bar{\omega}^{(k)}, \\
\partial_{n} \mathfrak{W}^{(k)} & =-\nabla K_{2}\left(\omega^{(k)}\right) \mathbf{n} & & \text { on } \Sigma^{(k)}, \\
\mathfrak{W}^{(k)} & =0 & & \text { on } \partial E,
\end{aligned}
$$

and $t^{(k)}$ is a positive scalar whose choice is to be discussed in Subsection 4.1. For the computation of $p$ and $p_{l}$, a data interpolation between $E \backslash \bar{\omega}$ and $\Omega \backslash \bar{\omega}$ is required.

\subsection{Numerical examples}

The state problem is discretized using standard triangular elements generated by the anisotropic mesh generator BAMG [12]. The location of the free boundary corresponding to a given inner boundary is not known a priori. However, when considering the situation where both $\omega$ and $\Gamma$ are concentric circles, then the location of the free boundary can be calculated analytically.

4.1.1 Example 1. We start with an example where the exact solution is known. Let $\mu=-1$, $\omega=B_{r_{1}}(0)$ and $\Omega=B_{C}(0)$. Then it is straightforwardly seen that the function

$$
u=-C \ln (r)+1+C \ln \left(r_{1}\right)
$$

satisfies $\Delta u=0$ in $\Omega \backslash \bar{\omega}, u=1$ when $r=r_{1}$, and $\nabla u \cdot \mathbf{n}=-1$ when $r=C$. Next, to solve the free boundary problem $\left(\mathcal{F}_{\omega}\right)$, we look for the value $C^{*}$ giving $u=0$ when $r=C^{*}$. For this, one needs to solve the equation

$$
-C \ln (C)+1+C \ln \left(r_{1}\right)=0,
$$

for the value of $C^{*}$. In what follows, we shall take $r_{1}=1$, in which case, $C^{*}$ is found to be $C^{*} \approx 1.76322$.

Therefore, by setting $E=B_{C^{*}}(0)$ where $\mu=-1$ in (1.4), we expect a circle of radius one to be the global minimizer of both $J_{1}$ and $J_{2}$, i.e.,

$$
E=\Omega_{T}:=\left\{(x, y) \in \mathbb{R}^{2}: x^{2}+y^{2}=\left(C^{*}\right)^{2}\right\} \quad \text { and } \quad \omega_{T}:=\left\{(x, y) \in \mathbb{R}^{2}: x^{2}+y^{2}=1\right\} .
$$

The optimization is performed using both cost functionals $K_{1}$ and $K_{2}$ starting from the same initial guess. The initial domains $\omega^{(0)}$ and $\Omega^{(0)}$ are given by

$$
\omega^{(0)}:=\left\{(x, y) \in \mathbb{R}^{2}: \frac{x^{2}}{1}+\frac{y^{2}}{1.2^{2}}=1\right\}, \quad \Omega^{(0)}:=\left\{(x, y) \in \mathbb{R}^{2}: x^{2}+y^{2}=4\right\},
$$




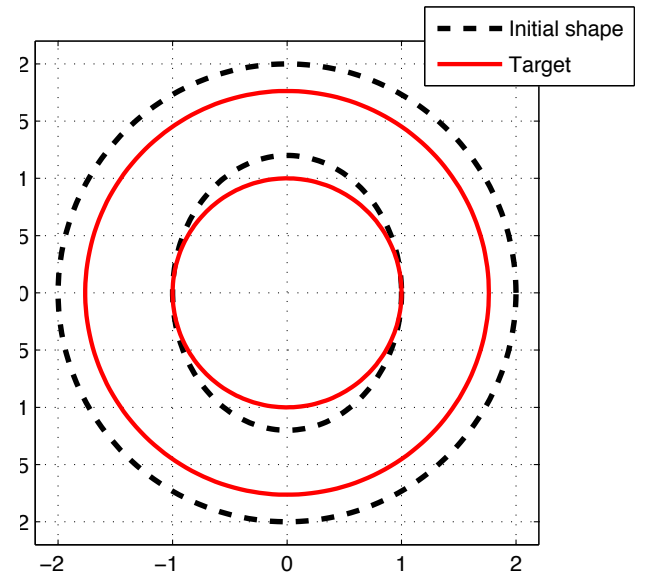

(a) Initial and target shape

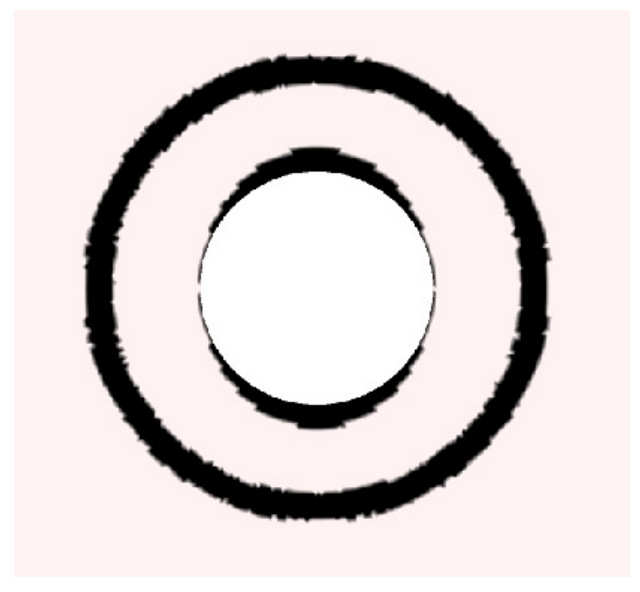

(b) Area to be minimized

FIG. 3. Initial shape $\Omega^{(0)}$ and target shape $E$

respectively. The boundaries $\Gamma^{(0)}, \Sigma^{(0)}$ and $\partial E, \Sigma_{T}$ of the initial and target shapes, respectively, are depicted in Figure 3a. In order to compute the value of $K_{1}$, the hold-all $\varepsilon:=[-4,4] \times[-4,4]$ embedding all admissible domains is utilized. The indicator function of the set $\left(\Omega \cap E^{c}\right) \cup\left(\Omega^{c} \cap E\right)$ is computed using

$$
\mathbb{1}_{\left(\Omega \cap E^{c}\right) \cup\left(\Omega^{c} \cap E\right)}(x)=\mathbb{1}_{\Omega}(x)+\mathbb{1}_{E}(x)-\mathbb{1}_{\Omega}(x) \mathbb{1}_{E}(x) .
$$

This indicator function, corresponding to the initialization in (4.10), is depicted in Figure 3b. For the initial value of the cost functional we get the numerical value $K_{1}\left(\omega^{(0)}\right) \approx 3.455$. Our aim is to minimize the area of the dark region in Figure $3 b$.

REMARK 4.1 The motion of $\Sigma$ is modeled explicitly using boundary nodes which are connected by line segments. These nodes are moved using the deformation field $\mathfrak{V}$ computed in (4.1-4.6). During each optimization step, the step size $t^{(k)}$ is chosen on the basis of the Armijo-type line search and such that there are no reversed triangles within the mesh after the update. If reversed triangles occur or the mesh quality deteriorates, then a new mesh is generated, see, e.g., [2, 24] for more details on mesh regeneration.

The parameters in $u_{a d}$ are set to:

$$
\omega_{\min }:=\left\{(x, y) \in \mathbb{R}^{2} \mid x^{2}+y^{2} \leqslant 0.61^{2}\right\}, \quad \omega_{\max }:=\left\{(x, y) \in \mathbb{R}^{2} \mid x^{2}+y^{2} \leqslant 1.75^{2}\right\} .
$$

We set the value of $t o l$ to $1 \times 10^{-3}$. After 28 iterations and no mesh regeneration, we reach the target shape (see Figure $4 \mathrm{a}$ ) with the final value $K_{1}\left(\omega^{\text {final }}\right) \approx 5.4 \times 10^{-3}$ for the cost. In Figure $4 \mathrm{~b}$, we depict the convergence history of $K_{1}$. From this figure, we observe that the cost is reduced during the optimization in a manner typical of gradient type methods, i.e., one observes a fast decrease in the beginning, and a slow convergence afterward. Moreover, since the target and final shapes of the boundaries practically coincide after optimization (see Figure 4a), the dark region is also minimized and tends to a set of measure zero. 


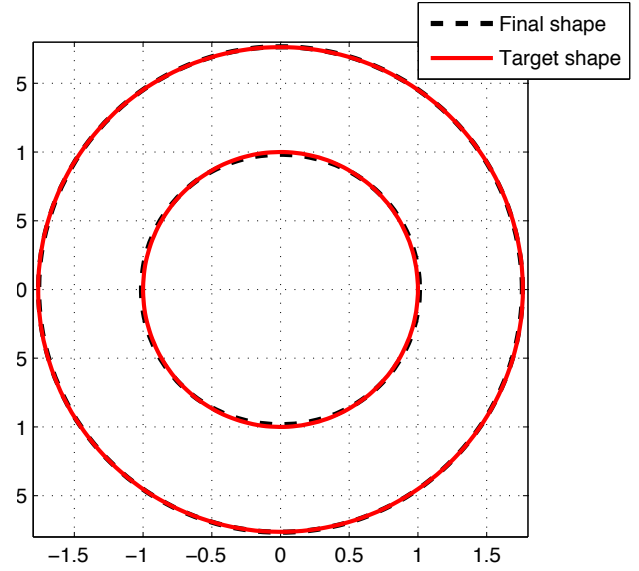

(a) Target and final shapes

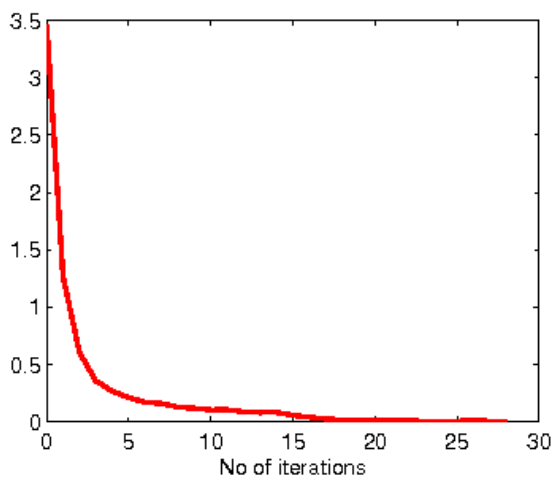

(b) History of $K_{1}$

FIG. 4. Final shape $\Omega^{\text {(final) }}$ and target shape using $K_{1}$

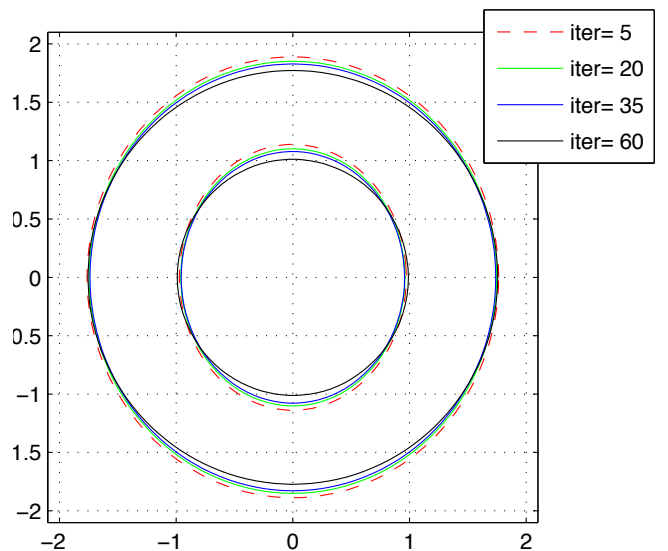

(a) Domain $\Omega^{*}(\omega) \backslash \bar{\omega}$

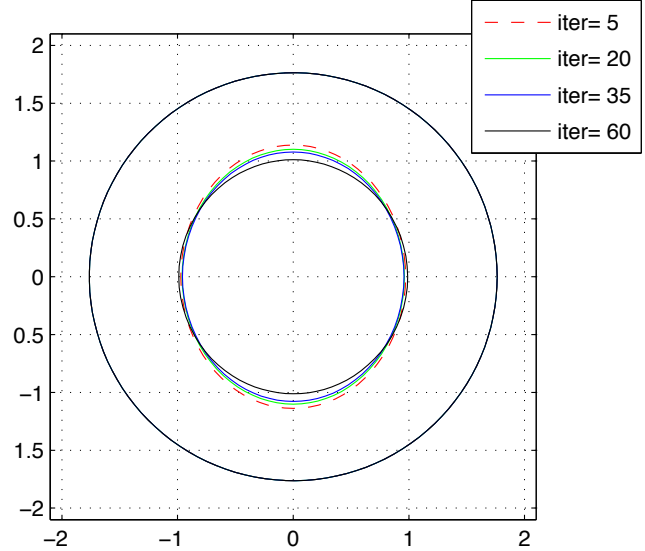

(b) Domain $E \backslash \bar{\omega}$

FIG. 5. Variations of domains $\Omega \backslash \bar{\omega}$ and $E \backslash \bar{\omega}$

Next, we perform the optimization using $K_{2}$. The initial value of the cost is found to be $K_{2}\left(\omega^{(0)}\right) \approx 0.036987$. In Figure 5, we plot the variations of the domains $\Omega^{*}(\omega) \backslash \bar{\omega}$ and $E \backslash \bar{\omega}$ with the iteration count. It is observed that as the iteration count increases, the boundary $\Sigma$ converges to the target (see Figure 5b). Similarly, the boundaries of $\Omega^{*}(\omega) \backslash \bar{\omega}$ converge to the target boundaries as well (see Figure 5a). The final boundaries are depicted in Figure 6a. As expected, the final shape coincides with the target shape.

The convergence history of $K_{2}$ is depicted in Figure 6b. After 60 iterations and no mesh regeneration, the value $K_{2}\left(\omega^{\text {final }}\right) \approx 2.527 \times 10^{-5}$ for the cost is obtained. 


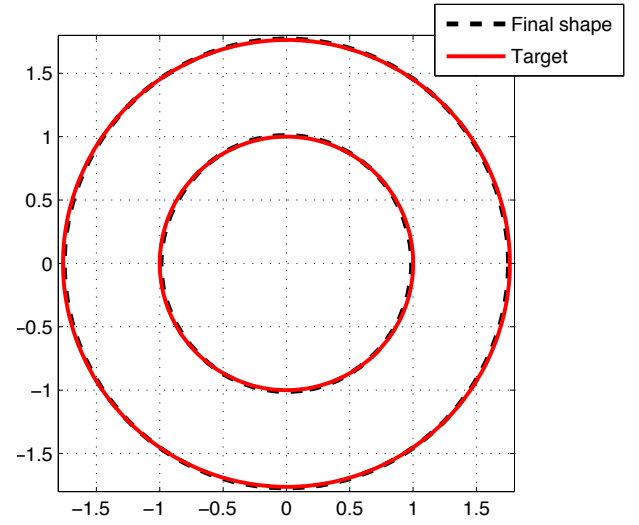

(a) Target and final shapes

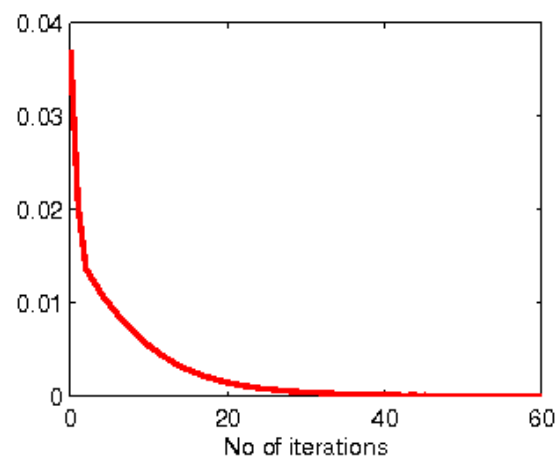

(b) History of $K_{2}$

FIG. 6. Final shape of the free boundary using $K_{2}$ and target shape

REMARK 4.2 In the numerical experiments we observe that the domains obtained using $K_{1}$ tend to have an oscillatory behaviour near the optimal shape, which is not the case with $K_{2}$. Therefore, in the subsequent examples, we use only $K_{2}$ which provides a more stable convergence.

4.1.2 Example 2. In this example our aim is to investigate the effect of increasing the value of $\mu$ on $\omega$ while the target boundary $\Gamma_{T}$ remains fixed. We set the target boundary as $\Gamma_{T}:=$ $\{r(t)(\cos 2 \pi t, \sin 2 \pi t) \mid t \in[0,1]\}$, where

$$
r(t)=0.5 \cos (2 \pi t)+0.8 \cos (4 \pi t)+2 .
$$

It is known that for the exterior Bernoulli free boundary problems with fixed inner component of the boundary, the respective free boundaries for $\mu \rightarrow 0^{-}$are asymptotic to a family of concentric circles with radii tending to infinity [9]. Therefore, one excepts the measure of the set $\Omega^{*}(\omega) \backslash \bar{\omega}$ to increase for $\mu \rightarrow 0^{-}$.

The initial design for $\Sigma$ is a circle of radius one while that of $\Gamma$ is a circle of radius $C$ (see Figure 7a). We choose $\mu=-3$ and discretize the initial domains $\Omega^{(0)} \backslash \bar{\omega}^{(0)}$ and $E \backslash \bar{\omega}^{(0)}$ with triangular elements. The boundary nodes of the triangulations of $\Omega^{(k)} \backslash \bar{\omega}^{(k)}$ and $E \backslash \bar{\omega}^{(k)}$ are used as the control parameters for the optimization. The free boundary problem (1.1)-(1.4) is computed and the initial value of the cost is $K_{2}\left(\omega^{(0)}\right) \approx 0.1071$. The parameters in $U_{\text {ad }}$ are set to:

$$
\omega_{\text {min }}:=\left\{(x, y) \in \mathbb{R}^{2} \mid x^{2}+y^{2} \leqslant 0.75^{2}\right\}, \quad \omega_{\max }:=\left\{(x, y) \in \mathbb{R}^{2} \mid x^{2}+y^{2} \leqslant 3.15^{2}\right\} .
$$

The final value of the cost $K_{2}$ after 111 optimization iterations and 7 mesh regenerations is found to be $6 \times 10^{-5}$. The optimal domain is depicted in Figure $7 \mathrm{~b}$. We observe that the final shape of $\Gamma^{*}(\omega)$ reaches almost perfectly the target boundary $\Gamma_{T}:=\partial E$. We compute the measure $\left|\Omega^{*}\left(\omega^{\text {final }}\right)\right\rangle$ $\bar{\omega}^{\text {final }} \mid \approx 4.36688$.

Next, we set $\mu=-1.8$ with an aim of checking whether the area of $\Omega^{*}(\omega) \backslash \bar{\omega}$ increases while $\Gamma^{*}(\omega)$ still coincides with the target $\Gamma_{T}$. We choose the same initialization as in Figure $7 \mathrm{a}$. The parameters in $U_{a d}$ are now set to:

$$
\omega_{\text {min }}:=\left\{(x, y) \in \mathbb{R}^{2} \mid x^{2}+y^{2} \leqslant 0.01^{2}\right\}, \quad \omega_{\max }:=\left\{(x, y) \in \mathbb{R}^{2} \mid x^{2}+y^{2} \leqslant 1.75^{2}\right\} .
$$




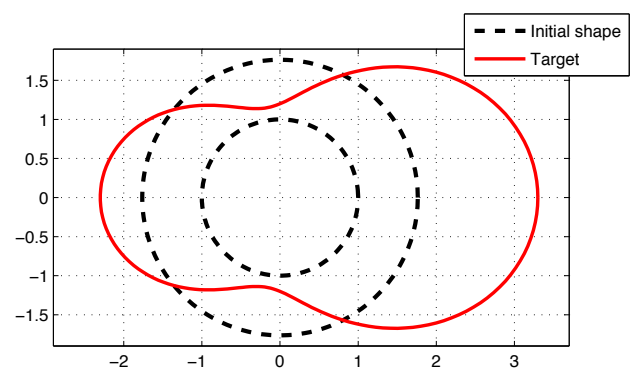

(a) Target and initial shape

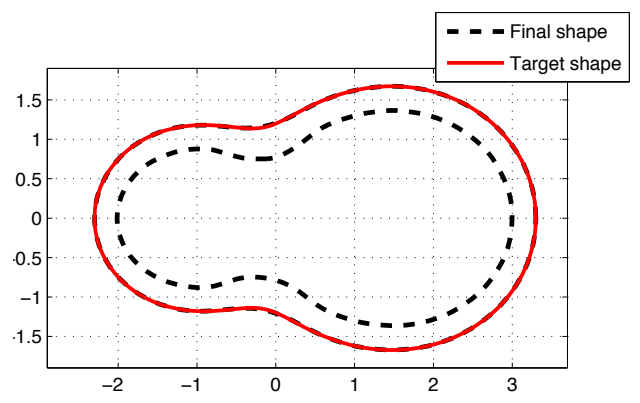

(b) Target and final shape

FIG. 7. Target $E$, initial $\omega^{(0)}, \Omega^{*}\left(\omega^{(0)}\right)$ and final shapes $\omega^{(\text {final })}, \Omega^{*}\left(\omega^{(\text {final })}\right)$ using $K_{2}$ with $\mu=-3$

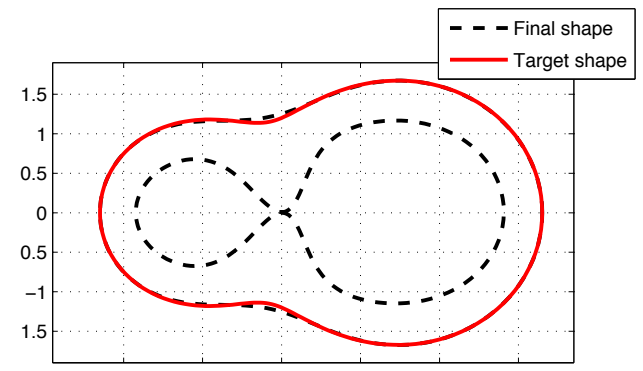

(a) Target and final shapes

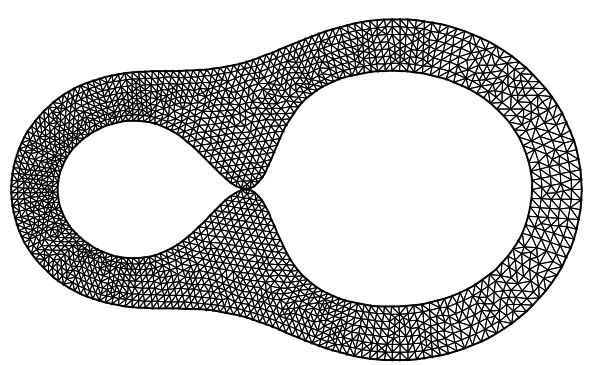

(b) Final mesh

FIG. 8. Target shape $E$ and final shapes $\omega^{(\text {final })}, \Omega^{*}\left(\omega^{(\text {final })}\right)$ using $K_{2}$ with $\mu=-1.8$

The initial value is $K_{2}\left(\omega^{(0)}\right) \approx 0.128$. After 120 iterations one observes that the boundary $\Sigma$ intersects itself at the origin (see Figure 8 ) and a meshing error occurs. At this point the optimization is stopped, the final value of $K_{2}\left(\omega^{\text {final }}\right) \approx 3.28 \times 10^{-4}$ is returned, and we compute $\left|\Omega^{*}\left(\omega^{\text {final }}\right)\right\rangle$ $\bar{\omega}^{\text {final }} \mid \approx 7.51295$. The kind of parametrization used here does not allow topological changes to occur during the optimization process. Therefore, we arrive at a similar conclusion as in [24], i.e., that the inner boundary consists of more than one connected component, although our numerical approach as well as the cost functionals $K_{1}$ and $K_{2}$ are different. Moreover, as expected, an increase in the value of $\mu$ for a fixed target, leads to an increase in the area of $\Omega^{*}(\omega) \backslash \bar{\omega}$, in agreement with the theory.

4.1.3 Example 3. In this example our aim is to check whether there exists a domain $\omega \in \boldsymbol{U}_{a d}$ such that $\Gamma^{*}(\omega)$ is as close as possible to a target $\Gamma_{T}$ which is not of class $\mathrm{C}^{\infty}$. We minimize $K_{2}$ with the boundary $\partial E$ of the target domain $E$ represented by a square with rounded corners (see Figure 9). The square is of dimension $[-2,2] \times[-2,2]$. Each of the corners is rounded using a quarter of a circle of radius one and centers $(1,-1),(1,1),(-1,1)$, and $(-1,-1)$, numbered counter-clockwise starting from corner $(2,-2)$. This target boundary can also be described using 
the parametric equations

$$
\begin{aligned}
& x(t)=2|\cos (2 \pi t)|^{\frac{1}{2}} \cdot \operatorname{sgn}(\cos (2 \pi t)), \quad t \in(0,1), \\
& y(t)=2|\sin (2 \pi t)|^{\frac{1}{2}} \cdot \operatorname{sgn}(\sin (2 \pi t)), \quad t \in(0,1) .
\end{aligned}
$$

With this parameterization, it is clear that the target is not of class $\mathrm{C}^{\infty}$. We set $\mu=-1$. The boundary $\Sigma$ is initialized using a circle of radius one while $\Gamma$ is initialized using a circle of radius $C$, both centered at the origin, cf. Figure 9.

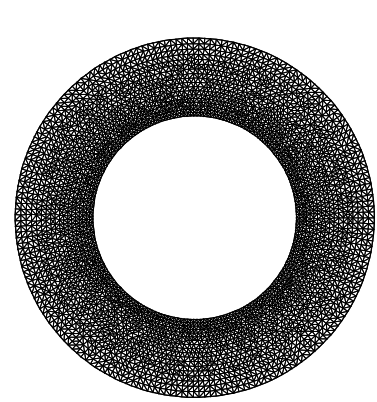

(a) $\Omega^{*}\left(\omega^{(0)}\right) \backslash \bar{\omega}^{(0)}$

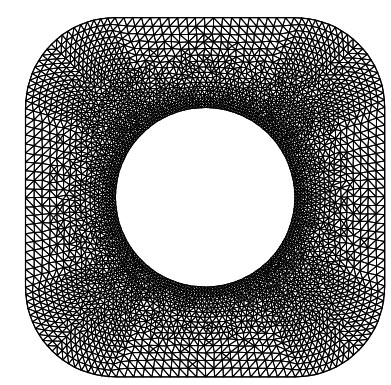

(b) Target $E \backslash \omega^{(0)}$

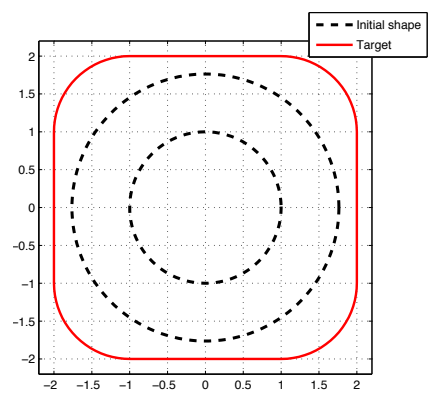

(c) Initial and target shapes

FIG. 9. Initial domains and target $E$

The parameters in $U_{a d}$ are set as in the first example. The initial value of the cost is $K_{2}\left(\omega^{(0)}\right) \approx$ 0.0954102. After 20 optimization steps and 5 remeshing, we obtain the final shape depicted in Figure $10 \mathrm{~b}$. We compute the final value $K_{2}\left(\omega^{(\text {final })}\right) \approx 1.1067 \times 10^{-3}$. In Figure 10 a, a comparison between the target outer boundary and the final outer boundary is made. We observe that the target is not reached exactly. In fact, some of the optimization variables attained the lower and upper bounds. Since the target is not of class $\mathrm{C}^{\infty}$, it cannot be reached using star-like boundaries $\Sigma$ of class $\mathcal{C}^{2}[1,24]$. The non-existence of $\omega \in U_{a d}$ such that $\Gamma^{*}(\omega)$ is as close as possible to the target $\Gamma_{T} \notin \mathrm{C}^{\infty}$ usually manifests itself through oscillations of $\omega$ [24]. However, since we use a regularized velocity field (see Algorithm 2), these oscillations of the inner boundary do not occur in our case, cf. [24, Example 2].

\section{Conclusions}

In this paper, we have performed the mathematical analysis for the sensitivity of a Bernoulli free boundary problem with respect to a shape perturbation of the inner boundary using the concepts of shape calculus. A new segregation algorithm for solving this free boundary PDE constrained shape optimization problem has been proposed and implemented. The numerical results presented here indicate that the derived shape gradients produce similar results as those obtained by Haslinger et al [24] using an automatic differentiation technique. The results in this paper can be extended to other bilevel problems and control of the free boundary in shape optimization.

Acknowledgments. The second author was supported in part by the Austrian Science Fund (FWF) under grant SFB F32 (SFB "Mathematical Optimization and Applications in Biomedical Sciences"). The third author acknowledges support from the DFG research center MATHEON (MATHEONProject C37 "Shape/Topology optimization methods for inverse problems"). 


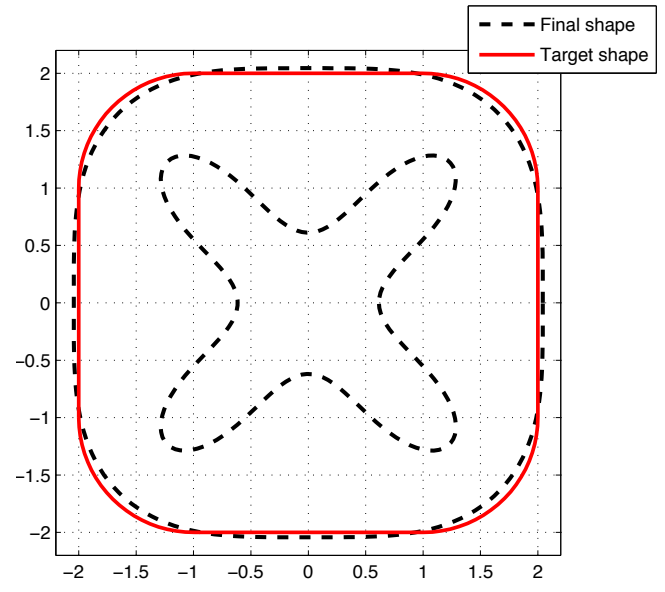

(a) Target shape and Final shape

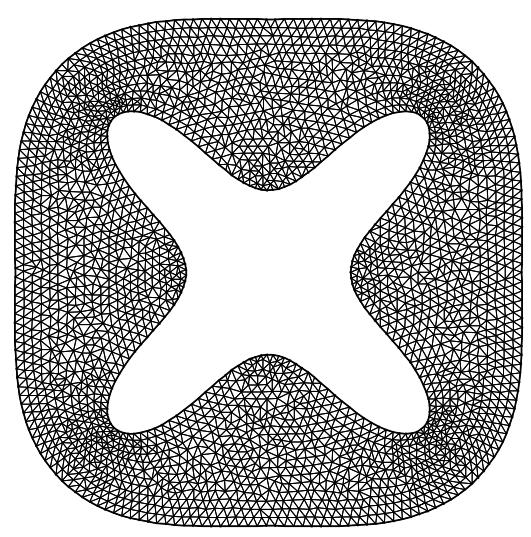

(b) Final mesh

FIG. 10. Initial and final shapes of the free boundary using $K_{2}$

\section{REFERENCES}

1. ACKer, A., \& MeYer, R., A free boundary problem for the p-laplacian: Uniqueness, convexity, and successive approximation of solutions. Electronic Journal of Differential Equations 19958 (1995), 1-20. Zb10820.35037 MR1334863

2. Allaire, G., \& Pantz, O., Shape optimization with freefem++. Structural and Multidisciplinary Optimization 32 (2006).

3. Bouchon, F., Clain, S., \& Touzani, R., Numerical solution of the free boundary Bernoulli problem using a level set formulation. Comput. Methods Appl. Mech. Engrg. 194 36-38 (2005), 3934-3948. Zb11090.76048 MR2149216

4. Bouchon, F., Clain, S., \& Touzani, R., A perturbation method for the numerical solution of the Bernoulli problem. J. Comput. Math. 261 (2008), 23-36. MR2378583

5. Bracken, J., \& MCGILl, J., Mathematical programs with optimization problems in the constraints. Operations Res. 21 (1973), 37-44. Mathematical programming and its applications. Zb10263.90029 MR0359797

6. Colson, B., Marcotte, P., \& SAVArd, G., Bilevel programming: a survey. 4OR 32 (2005), 87-107. Zbl1134.90482 MR2156141

7. Cuvelier, C., \& Schulkes, R. M. S. M., Some numerical methods for the computation of capillary free boundaries governed by the Navier-Stokes equations. SIAM Rev. 323 (1990), 355-423. Zbl0706. 76027 MR1069895

8. Delfour, M. C., \& Zolésio, J. P., Shapes and geometries: Analysis, differential calculus, and optimization. Society for Industrial and Applied Mathematics, Philadelphia, PA, USA, 2001. Zbl1002. 49029

9. Flucher, M., \& RumpF, M., Bernoulli's free boundary problem, qualitative theory and numerical approximation. J. Reine Angew. Math 486 (1997), 165-204. Zb10909. 35154 MR1450755

10. Haslinger, J., Ito, K., Kozubek, T., Kunisch, K., \& Peichl, G., On the shape derivative for problems of Bernoulli type. Interfaces and Free Boundaries 112 (2009), 317-330. Zbl1178.49055 MR2511644

11. Haslinger, J., \& MÄKInen, R. A. E., Introduction to Shape Optimization: Theory, Approximation, and 
Computation. Society for Industrial and Applied Mathematics, Philadelphia, PA, USA, 2003. Zbl1020. 74001 MR1969772

12. Неснт, F., Bamg: Bidimensional anisotropic mesh generator. http://www.rocq.inria.fr/gamma/ cdrom/www/bamg/eng.html, 1998.

13. Henrot, A., \& Pierre, M., Variation et optimisation de formes, vol. 48 of Mathématiques \& Applications (Berlin) [Mathematics \& Applications]. Springer, Berlin, 2005. Une analyse géométrique. [A geometric analysis]. Zbl1098.49001 MR2512810

14. Ito, K., Kunisch, K., \& Peichl, G., Variational approach to shape derivatives for a class of Bernoulli problems. Journal of Mathematical Analysis and Applications 3141 (2006), 126-149. Zbl1088.49028 MR2183542

15. Kasumba, H., \& Kunisch, K., Vortex control in channel flows using translational invariant cost functionals. Computational Optimization and Applications 52 (2012), 691-717. Zbl1258.49070 MR2950502

16. Kasumba, H., \& Kunisch, K., On free surface pde constrained shape optimization problems. Applied Mathematics and Computation 21823 (2012), 11429-11450. Zbl1278.49051 MR2943988

17. LAURAIN, A., \& Privat, Y., On a Bernoulli problem with geometric constraints. ESAIM Control Optim. Calc. Var. 181 (2012), 157-180. Zb106011582 MR2887931

18. Murat, F., \& Simon, J., Sur le contrôle par un domaine géométrique. Tech. rep., Publication du Laboratoire d'Analyse Numérique de l'Université Paris 6, 1976. 189 pages.

19. Panaras, G., Theodorakakos, A., \& Berggeles, G., Numerical investigation of the free surface in a continuous steel casting mold model. Metallurgical and Materials Transactions B 29 (1998), 1117-1126. 10.1007/s11663-998-0081-3.

20. Protter, M. H., \& Weinberger, H., Maximum principles in differential equations. Prentice-Hall Inc, Englewood Cliffs, N.J, 1967. Zb10153.13602 MR0219861

21. Selvanayagam, K., Götz, T., Sundar, S., \& Vetrivel, V., Optimal control of film casting processes. Int. J. Numer. Meth. Fluids 59 (2009), 1111-1124. Zbl1158.76324 MR2510941

22. Sokolowski, J., \& ZolÉsio, J. P., Introduction to Shape Optimization. Shape Sensitivity Analysis. Springer-Verlag, 1992. Zb10761.73003 MR1215733

23. Tilhonen, T., Shape optimization and trial methods for free boundary problems. Mathematical Modelling and Numerical Analysis 31 (1997), 805-825. Zbl0891.65131 MR1489173

24. Toivanen, J. I., Haslinger, J., \& MäKinen, R. A. E., Shape optimization of systems governed by Bernoulli free boundary problems. Computer Methods in Applied Mechanics and Engineering 19715 (2008), 3803-3815. Zbl1194.76031 MR2458115

25. Toivanen, J. I., Haslinger, J., \& MäKinen, R. A. E., Topology optimization in Bernoulli free boundary problems. Journal of Engineering Mathematics 80 (2013), 172-188. MR3055635

26. Troianiello, G. M., Elliptic differential equations and obstacle problems. The University Series in Mathematics. Plenum Press, New York, 1987. Zbl0655.35002 MR1094820

27. Volkov, O., Protas, B., Liao, W., \& Glander, D. W., Adjoint-based optimization of thermo-fluid phenomena in welding processes. J. Eng. Math 653 (2009), 201-220. Zbl1180 . 76050 MR2546438 